by Guifang Yang ${ }^{l}$, Zhenghong Chen $^{2}$, Mingzhong Tian ${ }^{l}$, Fadong $W^{l}$, Robert A.L. Wray, Yamin Ping ${ }^{l}$

\title{
On the growth of national geoparks in China: Distribution, interpretation, and regional comparison
}

1 School of Earth Sciences and Resources, China University of Geosciences, Beijing 100083, China. Email: yangcugb@gmail.com 2 Development Research Center, China Meteorological Administration, Beijing 100081, China

3 UOW College and School of Earth and Environmental Sciences, University of Wollongong, Wollongong 2522, Australia

Since the year 2000 China has created 139 National Geoparks; it started under the guidance of the former UNESCO's Division of Earth Science, and has therefore become one of the pioneers in this aspect. Many National Geoparks in China have been described over the past decade, but an understanding of the range of various landform features and their connection with geological and climatic constraints has not previously been published. Based on an increasing awareness of National Geoparks, the aim of this contribution is to provide a comprehensive overview of the National Geoparks of China by reviewing the geological heritage and their intrinsic linkages with geological and climatic controls. A regional comparison of the widespread clastic and karst Geopark landforms indicates that the development of these terrains can only be understood within a synthesis of tectonic constraints, climatic changes and lithological properties, whereas the variety of types, forms, scales and development patterns reflect processes in various climatic settings.

\section{Introduction}

A Geopark is a well-defined area that contains one or more geoheritage sites selected on the basis of scientific importance, rarity, scenic quality, or relation to geological history, events and processes (Eder and Patzak, 2004; UNESCO, 2008). The general perception over the past century or so has been that most geological features are highly resistant and thus do not require protection, and therefore geological surveys have been most commonly linked to the questions of mineral resource exploration and economic development. But as time has progressed, more attention is now being paid to preservation of sites of natural geoheritage, areas that in many cases demonstrate the real and nonrecurring nature of Earth's evolution (Leman et al, 2008). Establishment of new geoparks can also favor non-traditional economic development based upon geo-tourism and geo-products (Eder, 1999; UNESCO, 2008).
Since 1992, W. Eder (Director of UNESCO's Division of Earth Sciences) has argued for the designation of new "Geoparks" which focus on the sustainable utilization and conservation of geoheritage sites (Eder, 1999). As a continuation of earlier initiatives, and with support from UNESCO, the International Union of Geological Sciences (IUGS) began in 1995 to compile a global inventory of key geoscience localities and terrains (UNESCO, 156Ex/11Rev, 1999). In response to the development of the geopark concept the European Geopark Network was formed in 2000 (Zouros, 2002), followed in 2001 by the establishment of UNESCO Global Network of National Geoparks. In 2001 a meeting of interested scientists and policy makers at UNESCO's Paris headquarters took a substantial step forward in promoting the establishment of national or regional Geoparks in collaboration with UNESCO's Division of Earth Sciences. A further important milestone in the global geoparks movement came in 2004, when an initial Global Geoparks Network (GNN) was established on the basis of 25 geoparks in Europe and China. The GNN has now expanded to 67 Geoparks representing 25 countries from four different continents.

In parallel to the work of geoscientists in other countries, the geoscience community of China attaches great importance to the conservation of geosites, striving for the goal of scientific protection and sustainable development. The conservation of geological sites emerged in China in the 1980s, and has continued ever since in accordance with national laws and regulations, supported by government agencies, and promoted by nongovernmental organizations (Pan, 1995; Zhao and Zhao, 2003). The result of these efforts over the past two decades has been the designation of 139 conservation localities or districts (including the recently established Hong Kong National Geopark) as National Geoparks. Establishment of an additional 325 sites is currently in progress.

Despite their unique geological and scientific importance, the distribution pattern of many national geoparks within China remains poorly documented in the international literature (Zhao and Zhao, 2003; Leman et al., 2008). Reports of a relatively small number of Chinese National Geoparks with more spectacular features are widely scattered throughout the international earth sciences literature (such as karst landscapes in Guangxi of southeastern China or sandstone landforms in Zhangjiajie (forming some of the spectacular backdrops in the movie Avatar). But except for brief summaries by Zhao and Zhao (2003), Sun (2006), and Zhao and Zhao (2009) no 
comprehensive countrywide review of the diversity and range of Chinese geoparks have been published. The need for such a review is pressing, especially because the national geoparks that have already been designated cover a wide latitudinal and climatic ranges (Fig. 1).

The chief goal of this study is therefore to review the scattered reports of Chinese national geoparks to more clearly describe, firstly, the actual geographic locations of the geoparks, and secondly, the morphological and geological relationships between these Geoparks. On the basis of this we then assess and discuss the relative importance of lithological, tectonic and climatic controls in generating the diversity of landforms displayed in these Chinese geoparks. We finally combine geological and geographical information to attempt a systematic classification of the national geoparks of China.

\section{Materials and methods}

In order to meet the primary objectives of our present study we collected the relevant published references and reports (e.g., Wu, 2001; Zhao and Wang, 2002; Zhao and Zhao, 2003; Ministry of Land \& Resources of the People's Republic of China, 2004; Sun, 2006; Cui et al., 2007; Wei et al., 2008; Zhao and Zhao, 2009). We also incorporate available annual detailed reports of national geoparks and information provided on their websites (Liu, 1999; Li et al., 2002; $\mathrm{Wu}, 2005$; Peng, 2007). Various maps and figures were generated by superimposing the location of the geoparks on topographic and geologic maps. The complex geology and climatic patterns of the large Chinese landmass are summarized in the subsequent section, and provides the framework for the discussion and interpretation of the geoparks. Geoparks are then discussed on the basis of their relief, morphometric features and landform forming processes. In view of their abundance in China, and also the existing international reputation of some of these areas, selected karstic and clastic landforms are then discussed in more detail so as to verify the relative importance of lithologic and climatic constraints.

\section{Geoheritage resources in China}

China possesses numerous outstanding and varied geoheritage resources owing in large part to its unique 3-level stepwise geomorphic framework, with elevation descending from west to east (Fig. 1). Regrettably, until the 1980s there were no serious efforts in China to conserve geoheritage resources from unsustainable resource utilization and other intensive anthropogenic activities (Zhao and Wang, 2002). However in the past decade China has seen a rapid growth in interest and demand for geoheritage protection, and has embraced the international initiatives to develop the geopark concept. Expanding previously proposed classification systems (Zhao and Zhao, 2009), we propose a synthetic classification of the 139 national geoparks already established, and classify these geoheritage resources into 7 categories and numerous subcategories.

The most important of these geoheritage resources are diverse geomorphic features and landscapes, including karst, clastic, glacial, fluvial, volcanic and structural landforms. For centuries these spectacular landforms have attracted researchers of different backgrounds, as well as a large number of tourists from all over the world. Other important geoheritage resources include fossil beds, geohazard and mining sites, geological type-sections, and geological structural features. In order to systematize the description of these various types of resources and facilitate their preservation, the Ministry of Land and Resources of the People's Republic of China established in 2008 the classification system outlined in Table 1.

\section{Geological and climatic framework of National Geoparks in China}

\section{Tectonic controls}

China has a wide range of geological and tectonic settings, owing in large measure to its location on the southeastern edge of the Eurasia Plate, and adjoining the Pacific Plate and Gangdise - Indian plates (Wu, 2001). Many prior studies (Li et al., 2002; Ministry of Land and Resources of the People's Republic of China, 2004) have shown that the major fault and fracture systems in the region exert a dominant control on landform development, via differential uplift and widening of fractures and joint sets. The basement structure of most of southern China was established by the Wuling-Xuefeng Orogenic Movement ( 1400-850 Ma), and during the Late Triassic Indosinian Movement China underwent a complex process of NW-SE extrusion and eastwest shearing, producing associated groups of NW-NNW tensionfaults and NS and NNW strike-slip faults. The early Yanshanian Movement during the late Jurassic period (around 160-140 Ma), caused EW extrusion of China and formation of NNE-NS folds together with two sets of strike-slip faults in NNW and NEE directions. The late Yanshanian Movement of late Cretaceous age (around 130 68 $\mathrm{Ma}$ ), displaced south China from SE to NW, with reactivation and enlargement of faults, uplift and tilting (Wu, 2001).

Juxtaposition of diverse geological features provides an ideal basis for the occurrence of a variety of landscapes (Fig.2). In China landforms deemed worthy of national geopark status tend to be concentrated along the discontinuities that mark the boundary zones between the three topographic benches that characterize Chinese physiography (Fig. 2). These boundaries are areas of intense tectonic activity. For example, the huge nappes of the Longmen Mountains (Geopark 54), the waves in a river caused by rapids, or undulating landscape in the Dadu Valley (Geopark 59), and the gushing hot springs and volcanic landscapes in Tengchong (Geopark 125; Fig. $3 \mathrm{j}$ ) are all found along the westernmost and highest geomorphic step. Extensive differential uplift and subsidence along pediment faults along the second geomorphic step has given rise to the impressive red cliffs and waterfalls in the grand Taihang, Wuling, and Nanling mountains (Figs. 4d, 4e, 4f, 4g, 4h). The third, easternmost and lowest, geomorphic step includes terrains corresponding to the Circum-Pacific volcanic belt and is characterized by steep mountains and towering crags, such as those at Yandang Mountain of Zhejiang(Figs. 5f, 5g), Taimushan (Fig. 5h) and Zhangzhou of Fujian (Geopark 103), and Huguangyan of Guangdong (Geopark 136).

Differential tectonic uplift and subsidence in Mesozoic and Cenozoic times has also given rise to many unique local landforms, such as the remarkable hoodoos in Yunnan, the secluded mountain landscapes in Jiangxi, the majestic and beautiful Huangshan landforms in Anhui, and the precipitous Songshan terrain in Henan. Intersecting joint systems have provided structural control for fluvial incision and glacial erosion, giving rise to the striking pinnacles and peak clusters, narrow ridges and deep gullies in these areas.

The diversity of landforms also reflects the interplay of tectonic and lithologic constraints. For instance, the stepped landforms at Zhangjiajie (Fig. 4f) that are developed on flat-lying intercalated Devonian sandstone and siltstones have been affected by intermittent 


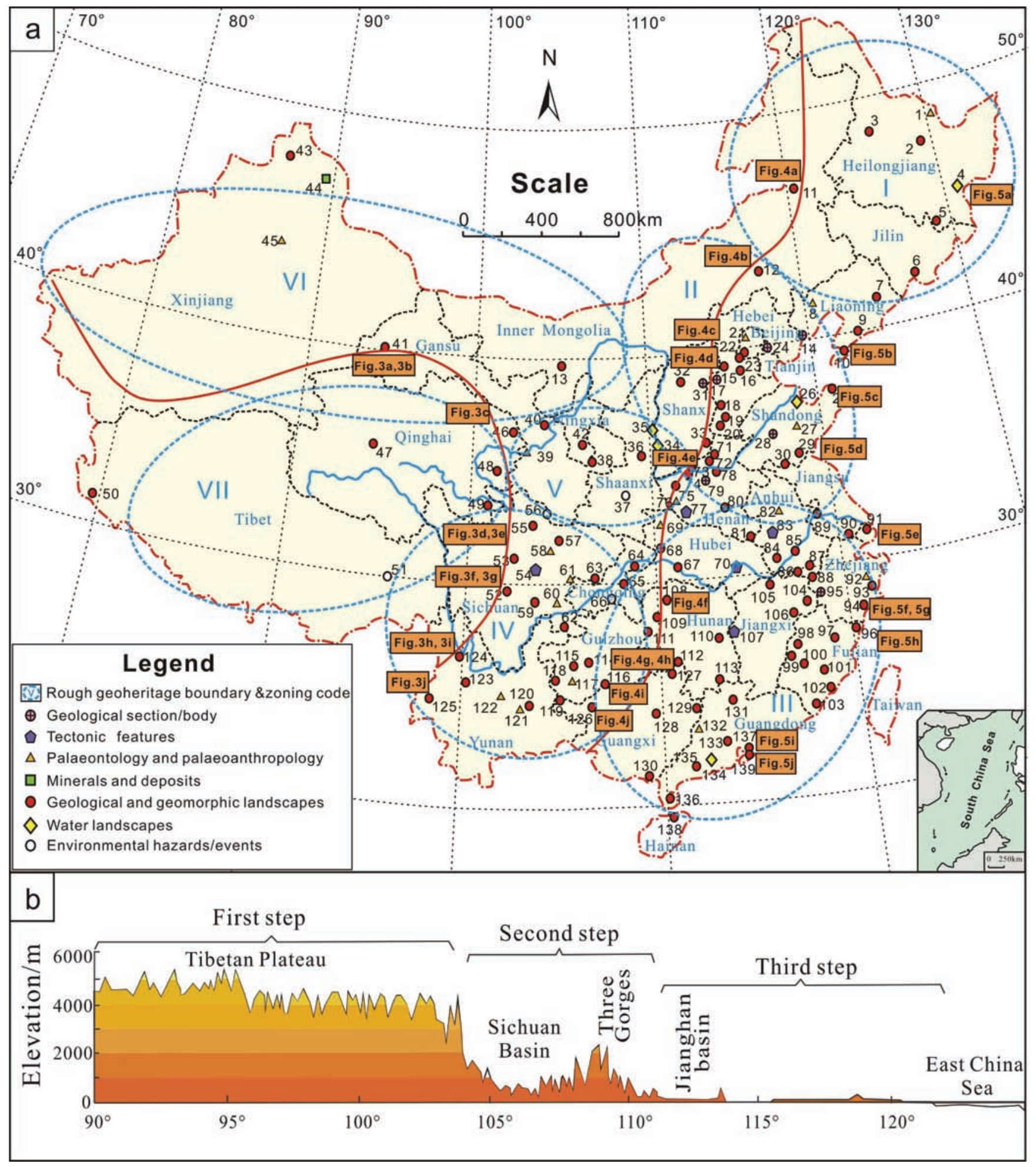

Figure 1. (a) Sketch map of China indicating the localities of national geoparks; (b) Three major geomorphic levels of China.

Cenozoic uplift processes (Yang et al., 2011). The uplands are deeply dissected and the entire massif has been uplifted 300-500 $\mathrm{m}$ along the marginal faults. This has given rise to extensive flat surfaces often separated by steep cliffs, shaped mainly by scarp retreat caused by fluvial sapping and mass failure. Overall, the larger structural lineaments at Zhangjiajie control the major streams and escarpments, but more importantly, the smaller fractures and joints break the rock mass into smaller-size blocks. Thus, whilst the major drainage patterns follow first-order tectonic controls, the spectacular topographic features at this and other geoparks were shaped by the smaller-scale but more densely distributed joints.

Similarly, temporally-variable eruptions have given rise to volcanic rock sequences with variable lithologic characteristics, as evidenced for instance in Zhangzhou National Geopark, and Arxan National Geopark (Fig. 4a). As part of the Circum-Pacific belt, the peri-Pacific region in the stable platform area of eastern China has 


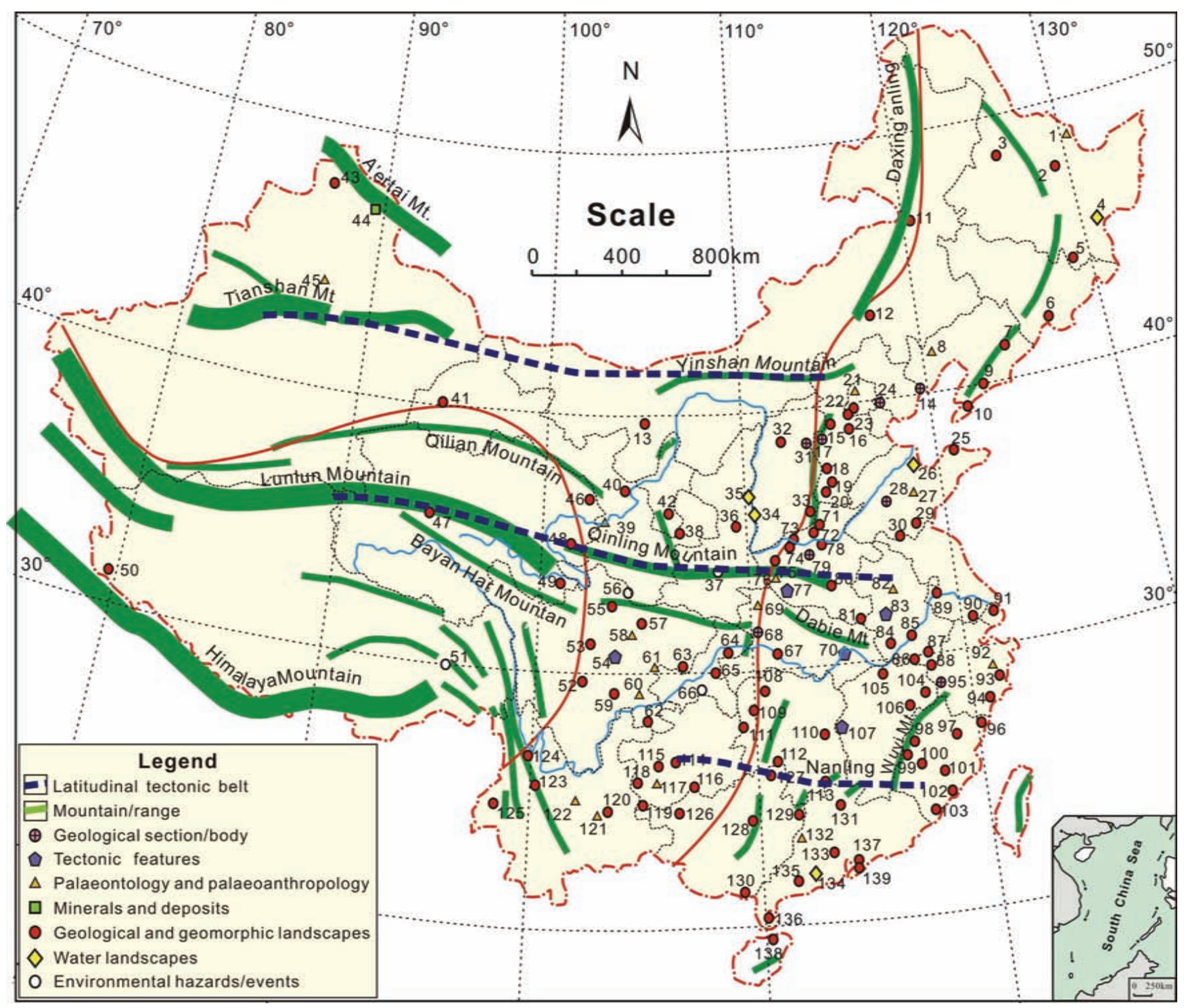

Figure 2. Distribution pattern of Chinese mountain ranges and style of geoparks.

experienced four episodes of volcanic activity since the Cenozoic (and, locally, late Mesozoic), forming the 'East Asia continental rift belt' (Liu, 1999). The National Geoparks Wudalianchi in Heilongjiang, Fushan in Anhui, Linhai in Zhejiang, Zhangzhou in Fujian, and Huguangyan in Guangdong each display typical characteristics of variable volcanic sequences controlled by their different tectonic settings within the East Asia continental rift belt.

Mass failure triggered by earthquakes or other abrupt geological events is also important in shaping the landforms of tectonically active zones (Pan, 1995). For example, earthquakes have triggered landslides over large areas, and this has been the case in Cuihuashan, Yigong Geopark in Tibet, and Zigong in Sichuan (Fig. 1 and Table 1). However even in presently tectonically quiescent regions, there may be topographic features of past earthquake activity such as extensive relict collapse deposits.

\section{Climatic zonation}

Many previous studies (Pan, 1995; Zhao and Zhao, 2003; $\mathrm{Wu}, 2005$ ) have shown the importance of the large range of climate types in China (Fig. 6) in determining the style of denudation and morphology. Nationally-averaged annual rainfall is $630 \mathrm{~mm}$ per year and decreases from the southeast coastal areas towards the northwest inland region. The southeast coastal areas of Guangdong, Guangxi, eastern Fujian, Jiangxi, Zhejiang and Taiwan are characterized by a tropical to subtropical monsoon climate, with relatively high annual precipitation of 1500-2000 mm. Annual rainfall in the Yangtze River basin, the Huaihe River basin and Qinling area are approximately $1000 \mathrm{~mm}, 800 \mathrm{~mm}$ and $800 \mathrm{~mm}$, respectively. In the Lower Yellow River basin, Weihe River basin, Haihe River basin and most parts of northeast Daxinganling rainfall is around $500-750 \mathrm{~mm}$, and, in contrast, the annual rainfall in the northwest inland region is only 100 200 mm. Further west, the extremely dry Tarim, Turpan and Qaidam Basins of Xinjiang receive less than $50 \mathrm{~mm}$, with the central parts of these hyper-arid basins receiving less than $20 \mathrm{~mm}$ annually. Temperatures show a similar trend, decreasing from an average of $>20^{\circ} \mathrm{C}$ in the southeast coastal areas towards the much colder northwest China. The coldest areas are located in the Qinghai-Tibetan Plateau in the west and in the extreme northeast China, where annual temperatures are $<-4^{\circ} \mathrm{C}$. 
Table 1. National geoparks in China classified by major geoheritage feature or value

\begin{tabular}{|c|c|c|}
\hline Classification & Representative national geoparks (number) & Major features of geoheritage resources \\
\hline $\begin{array}{l}\text { A: Geological } \\
\text { sections and } \\
\text { geological bodies }\end{array}$ & $\begin{array}{l}\text { Liujiang National Geopark, Qinhuangdao (14) } \\
\text { Fuping Natural Bridge National Geopark (17) } \\
\text { Jixian National Geopark (24) } \\
\text { Taishan National Geopark (28) } \\
\text { Wutaishan National Geopark (31) } \\
\text { Shennongjia National Geopark (68) } \\
\\
\text { Songshan National Geopark (79) } \\
\text { Changshan National Geopark (95) }\end{array}$ & $\begin{array}{l}\text { Complete stratigraphic section of North China; marine erosion landforms; } \\
\text { granite landforms } \\
\text { Archeozoic standard Longquanguan Formation } \\
\text { Middle-Upper Proterozoic stratigraphic section } \\
\text { Stratigraphic sections of Neoarcheozoic to PaleoPreterozoic, Cambrian and } \\
\text { Early Ordovician; Early Paleozoic fossils; neotectonic landforms } \\
\text { Wutai Group, Hutuo Group, Wutai tectonic movement; periglacial landforms } \\
\text { Folded metamorphic basement; Pleistocene palaeoanthropic relics, } \\
\text { mountainous glacial, fluvial and karst landforms } \\
\text { Late Archean-Early Proterozoic stratigrapohic unit } \\
\text { GSSP stratotype profile of Darriwilian Stage reef limestones }\end{array}$ \\
\hline $\begin{array}{l}\text { B: Structural } \\
\text { features at } \\
\text { different scales }\end{array}$ & $\begin{array}{l}\text { Longmenshan National Geopark (54) } \\
\text { Mulanshan National Geopark, Wuhan (70) } \\
\text { Baotianman National Geopark, Neixiang (77) } \\
\text { Dabieshan (Liu'an) National Geopark (83) } \\
\text { Wugongshan National Geopark (107) }\end{array}$ & $\begin{array}{l}\text { Long-distance nappe structure/Longmenshan tectonic zone } \\
\text { Plate collisional high-pressure belt; Blueschist of metamorphic belt } \\
\text { Tectonism; metamorphism; granite forest landforms; Proterozoic-Paleozoic } \\
\text { strata } \\
\text { Stylolite structure; ultra-high pressure metamorphic zone; granulite-facies } \\
\text { rocks; granite peak clusters; caves; canyons; volcanic landforms; silicified } \\
\text { wood and neotectonism } \\
\text { Structures of metamorphic core complexes; granite peaks and cliffs }\end{array}$ \\
\hline \multirow[t]{4}{*}{$\begin{array}{l}\text { C: Palaeontology } \\
\text { and palaeo- } \\
\text { anthropology }\end{array}$} & $\begin{array}{l}\text { C-1: Palaeoanthropology } \\
\text { Fengkai National Geopark (132) }\end{array}$ & $\begin{array}{l}\text { Relics and fossils of ancient human-beings; karst landforms; fluvial } \\
\text { landforms }\end{array}$ \\
\hline & $\begin{array}{l}\text { C-2: Palaeoanimals } \\
\text { Dinosaur } \\
\text { Jiayin Dinosaur National Geopark (1) } \\
\text { Liujiaxia Dinosaur National Geopark (39) } \\
\text { Zigong Dinosaur and Palaeoorganism } \\
\text { National Geopark (60) } \\
\text { Yunxian Dinosaur-Egg Fossil Cluster National } \\
\text { Geopark (69) } \\
\text { Funiushan National Geopark, Xixia (76) } \\
\text { Lufeng Dinosaur National Geopark (122) }\end{array}$ & $\begin{array}{l}\text { Dinosaur fossils of Late Cretaceous } \\
\text { Larger dinosaur footprints (with an diameter of }>1 \mathrm{~m} \text { ) } \\
\text { Various dinosaur fossils; complete dinosaur skeletons; dinosaur skins } \\
\text { Cretaceous dinosaur-egg fossils; Australopithecus; paleoanthropology } \\
\text { Centralized dinosaur-egg fossils; Qinling orogenic belt } \\
\text { Middle-Jurassic dinosaur fauna; Late Late-Miocene Australopithecus; } \\
\text { Palaeomining sites }\end{array}$ \\
\hline & \begin{tabular}{|l|} 
Mixed palaeofauna \\
Chaoyang Bird Fossil National Geopark (8) \\
Shanwang National Geopark (27) \\
Anxian Bioherm National Geopark (58) \\
Bagongshan National Geopark, Huainan (82) \\
Guanling Fossil Cluster National Geopark (117) \\
Chenjiang Ancient Fauna National Geopark (121)
\end{tabular} & $\begin{array}{l}\text { Jehol fauna with abundant birds, dinosaurs, primitive mammals, earliest } \\
\text { flowers, and diverse insects } \\
\text { Abundant and perfectly preserved Miocene biota } \\
\text { Devonian siliceous sponge reef } \\
\text { Huainan biota }(0.7-0.8 \mathrm{Ga}) \text {; Precambrian-Cambrian stratigraphic section } \\
\text { Late Triassic marine reptile and crinoids fossils showing eco- } \\
\text { environment and palaeotaphonomy } \\
\text { Early Cambrian }(0.7-0.8 \mathrm{Ga}) \text { Explosion with simultaneous emergence } \\
\text { of dozens of biological categories }\end{array}$ \\
\hline & $\begin{array}{l}\text { C-3: Palaeobotany } \\
\text { Yanqing Silicified Wood National Geopark (21) } \\
\text { Qitai Silicified Wood-Dinosaur National } \\
\text { Geopark (45) } \\
\text { Shehong Silicified Wood National Geopark (61) } \\
\text { Xinchang Silicified Wood National Geopark (92) }\end{array}$ & $\begin{array}{l}\text { Clusters of in-situ buried silicified wood } \\
\text { Silicified wood fossils; dinosaur fossils; yardang landform } \\
\text { Silicified wood and palaeotological fossils } \\
\text { Silicified wood of Cretaceous; Danxia landforms }\end{array}$ \\
\hline $\begin{array}{l}\text { D: Minerals and } \\
\text { ore deposits }\end{array}$ & Koktokay National Geopark, Fuyun (44) & $\begin{array}{l}\text { Relics of mining for granite pegmatite-type rare metal deposit; } \\
\text { earthquake landforms; granite landscapes }\end{array}$ \\
\hline $\begin{array}{l}\text { E: Geomorphic } \\
\text { features and } \\
\text { landscapes }\end{array}$ & $\begin{array}{l}\text { E-1: Rock landscapes } \\
\text { Granite landforms } \\
\text { Yichun Granite Stone-Forest National } \\
\text { Geopark (2) } \\
\text { Hexigten National Geopark (12) } \\
\text { Yimengshan National Geopark (29) }\end{array}$ & $\begin{array}{l}\text { Granite peak clusters, forests and pillars; Indosinian granites with } \\
\text { fractures and joints; gravity collapse } \\
\text { Granites with horizontal joints; man-like and objective-like granite pillars } \\
\text { Granite peak clusters and forests; gems and jades }\end{array}$ \\
\hline
\end{tabular}


Table 1. National geoparks in China classified by major geoheritage feature or value

\begin{tabular}{|c|c|c|}
\hline Classification & Representative national geoparks (number) & Major features of geoheritage resources \\
\hline & $\begin{array}{l}\text { Shenlingzhai National Geopark, Luoning (75) } \\
\text { Chayashan National Geopark (80) } \\
\text { Tianzhushan National Geopark (84) } \\
\text { Guniujiang National Geopark, Qimen (86) } \\
\text { Huangshan National Geopark (87) } \\
\text { Taimushan National Geopark, Fuding (96) } \\
\text { Shiniushan National Geopark, Dehua (101) } \\
\text { Sanqingshan National Geopark (104) }\end{array}$ & $\begin{array}{l}\text { Granite landscapes; cone-shaped peaks; spherical weathering } \\
\text { Granite peak forests and stone egg landforms; gravity collapse; } \\
\text { fluvial erosion } \\
\text { Granite peak clusters; ultra-high pressure metamorphic belt; Quaternary } \\
\text { mammal fossils } \\
\text { Granite peak clusters and forests; waterfalls } \\
\text { Granite peak forest; rock pillars; grotesque peaks; queer rocks; deep and } \\
\text { secluded canyons; warm springs; spectacular pines } \\
\text { Granite with potassic vugs and fissures; granite hoodoos; peak cluster and } \\
\text { cavities; gravity collapse; marine erosion landforms } \\
\text { Granite peak clusters; volcanic landforms } \\
\text { Granite peak forests and clusters; stacked caves }\end{array}$ \\
\hline & $\begin{array}{l}\text { Clastic landforms } \\
\text { Bingyugou National Geopark, Dalian (9) } \\
\text { Zhangshiyan National Geopark, Zanhuang (18) } \\
\text { Wu'an National Geopark (20) } \\
\text { Huguan Canyon National Geopark (33) } \\
\text { Kongtongshan National Geopark, Pingliang (38) } \\
\text { Yellow River Stone Forest National Geopark, } \\
\text { Jingtai (40) } \\
\text { Huoshizhai National Geopark, Xiji (42) } \\
\text { Kanbula National Geopark, Jainca (48) } \\
\text { Jiangyou National Geopark (57) } \\
\text { Guanshan National Geopark (71) } \\
\text { Yuntai National Geopark, Jiaozuo (72) } \\
\text { Wangwushan National Geopark (73) } \\
\text { Daimeishan National Geopark, Luoyang (74) } \\
\text { Qiyunshan National Geopark (88) } \\
\text { Taining National Geopark (98) } \\
\text { Yong'an National Geopark (100) } \\
\text { Longhushan Danxia Geomorphy National } \\
\text { Geopark (106) } \\
\text { Zhangjiajie Sandstone Peak Forest National } \\
\text { Geopark (108) } \\
\text { Langshan National Geopark (112) } \\
\text { Feitianshan National Geopark, Chenzhou (113) } \\
\text { Liming-Laojunshan National Geopark, } \\
\text { Yulong (124) } \\
\text { Ziyuan National Geopark (127) } \\
\text { Danxiashan National Geopark (131) }\end{array}$ & $\begin{array}{l}\text { Sandstone peak clusters; faults and joints; Pleistocene valley glaciers } \\
\text { Red cliffs and long walls; sedimentary profiles and bedding structures; } \\
\text { Valleys; peak clusters; stepped landforms; basalt eruption } \\
\text { Stepped valleys; water bodies; barrier valleys } \\
\text { Danxia landforms; structures formed in Himalayan movement } \\
\text { Stone forest landforms of glutenite; Yellow River valley; neotectonism } \\
\text { Danxia landform and Loess Plateau } \\
\text { Danxia landform; sedimentary sequence and fluvial terraces since } \\
\text { Tertiary; periglacial landforms } \\
\text { Danxia-type landforms; karst cave and collapses; standard Devonian } \\
\text { stratigraphic profiles } \\
\text { Cliffs; peak forest; peak clusters; valleys } \\
\text { Canyons; valleys; gorges; red cliffs and long walls; residual hills; } \\
\text { waterfalls; karst caves } \\
\text { Red cliffs and long walls; valleys; unconformity and ancient strata; } \\
\text { ancient and modern water conservancy projects } \\
\text { Stepped valleys; peak clusters; water landforms; shallow sedimentary } \\
\text { structures } \\
\text { Danxia landform; red cliffs and lone walls; flat caves; natural bridges; } \\
\text { dinosaur fossils } \\
\text { Various peak pillars, prisms, precipices, and overhanging rocked } \\
\text { mirrored on lake; queer rocks; caves; valleys } \\
\text { Danxia landform; karst landforms; typical stratigraphic profiles } \\
\text { Danxia landform with grotesquely shaped rocks and hills } \\
\text { Quartz sandstone landforms with mesa, square mountain, peak clusters } \\
\text { and forests; pillars; valleys; karst caves } \\
\text { Danxia landform; tafelbergs, peak pillars, canyons and precipices } \\
\text { Danxia landform; cavities, valleys, natural bridges, cliffs } \\
\text { Alpine Danxia landform; glacial relics; modern glacial valleys } \\
\text { Danxia landform; tafelbergs, peak pillars and precipices } \\
\text { Danxia landform, where this typical landform is named }\end{array}$ \\
\hline & $\begin{array}{l}\text { Karst landforms } \\
\text { Benxi National Geopark (7) } \\
\text { Baishishan National Geopark, Laiyuan (15) } \\
\text { Yeshanpo National Geopark, Laishui (16) } \\
\text { Lincheng National Geopark (19) } \\
\text { Shihuadong National Geopark (22) } \\
\text { Shidu National Geopark (23) } \\
\text { Xiong'ershan-Baodugu National Geopark (30) } \\
\text { Jiading National Geopark, Huzhu (46) } \\
\text { Huanglong National Geopark (55) } \\
\text { Xingwen Stone Forest National Geopark (62) }\end{array}$ & $\begin{array}{l}\text { Karst depressions and dolines; caves; underground streams; standard } \\
\text { section of north China } \\
\text { Dolomite peaks, columns, and cliffs; springs; source of Laishui River } \\
\text { Karst valleys; peak clusters; granite peaks and cliffs; caves } \\
\text { Karst caves and stepped landfroms in canyons } \\
\text { Limestone karst caves with numerous stalactites, stone flowers, prisms, } \\
\text { stalagmites, underground streams } \\
\text { Karst valleys; peak clusters; caves } \\
\text { Karst hills with spectacular shapes; queer valleys; singular caves } \\
\text { Karst landscapes; glacial landforms; Danxia landforms; canyons } \\
\text { Travertines; waterfalls; rimstone dams; colorful pools; travertine beach } \\
\text { Karst peak clusters; hoodoos; huge cavities; big deep dolinen; valleys } \\
\text { and waterfalls }\end{array}$ \\
\hline
\end{tabular}


Table 1. National geoparks in China classified by major geoheritage feature or value

\begin{tabular}{|c|c|c|}
\hline Classification & Representative national geoparks (number) & Major features of geoheritage resources \\
\hline & $\begin{array}{l}\text { Huayingshan National Geopark (63) } \\
\text { Longgang National Geopark, Yunyang (64) } \\
\text { Wulong Karst National Geopark (65) } \\
\text { Xishan National Geopark at Lake Taihu, } \\
\text { Suzhou (90) } \\
\text { Tian'e Caves National Geopark, Ninghua (99) } \\
\text { Guzhang Red Carbonate-Rock Stone Forest } \\
\text { National Geopark (109) } \\
\text { Jiubujiang National Geopark (110) } \\
\text { Fenghuang National Geopark (111) } \\
\text { Shuanghedong National Geopark, Suiyang (114) } \\
\text { Zhijindong National Geopark (115) } \\
\text { Pingtang National Geopark (116) } \\
\text { Wumengshan National Geopark, Liupanshui (118) } \\
\text { Xingyi National Geopark (119) } \\
\text { Shilin Karst Peak Forest National Geopark (120) } \\
\text { Dashiwei Karst National Geopark in Leye, } \\
\text { Baise (126) } \\
\text { Xiangqiao Karst National Geopark, Luzhai (128) } \\
\text { Fengshan Karst National Geopark (129) } \\
\text { Lingxiaoyan National Geopark, Yangchun (135) }\end{array}$ & $\begin{array}{l}\text { Middle-lower karst mountains; geological tectonics; stratigraphic } \\
\text { sections; terrestrial heat } \\
\text { Big deep dolines; caves; canyons } \\
\text { Karst landforms; hoodoos; canyons; natural bridges; caves; deep dolines } \\
\text { Karst caves; lake erosion landforms; tectonic landforms } \\
\text { Karst caves with Cretaceous fish fossils, Pleistocene vertebrate } \\
\text { fossils and palaeoanthropologic relics } \\
\text { Red carbonate hoodoos; karst landscapes; canyons; caves } \\
\text { Karst peak clusters; canyons; caves; natural bridges; waterfalls; lakes; } \\
\text { fossils sites } \\
\text { Karst canyons; peak forests; tablelands; karst caves; waterfalls } \\
\text { Gypsum crystal flowers in dolomites; warm springs } \\
\text { Karst clusters and forests; buttes; canyons; karst lakes; cave deposits } \\
\text { Plateau karst landforms; canyons; deep caves } \\
\text { Karst caves and canyons; plateau karst landforms; fossils sites and } \\
\text { palaeoanthropogenic relics } \\
\text { Karst peak clusters and forests; valleys; waterfalls; Keichousaurus hui fossils } \\
\text { Karst hoodoos; sword-shaped pinnacles; peak clusters; caves; waterfalls } \\
\text { Funnels, valleys, peak clusters, and natural bridges formed by } \\
\text { underground streams; cave deposits } \\
\text { Karst landforms; chick-like peak clusters and forests; natural bridge; } \\
\text { waterfalls } \\
\text { Karst landforms with high peak forests and deep depressions } \\
\text { Karst landforms and caves; underground river; palaeoanthropologic } \\
\text { relics; peak flying from afar }\end{array}$ \\
\hline & $\begin{array}{l}\text { Loess landforms } \\
\text { Luochuan Loess National Geopark (36) }\end{array}$ & Loess landforms; standard loess section; loess landforms \\
\hline & $\begin{array}{l}\text { Aeolian landforms } \\
\text { Alxa Desert National Geopark (13) } \\
\text { Yardang National Geopark (41) } \\
\text { Zanda Clay Forest National Geopark (50) }\end{array}$ & $\begin{array}{l}\text { Deserts and embedded lakes; aeolian landforms } \\
\text { Grotesquely shaped landforms } \\
\text { Soil forest landforms }\end{array}$ \\
\hline & $\begin{array}{l}\text { E-2: Volcanic landforms } \\
\text { Wudalianchi Volcanic National Geopark (3) } \\
\text { Jingpohu National Geopark (5) } \\
\text { Jingyu Volcano and Warm Mineral Spring } \\
\text { Cluster National Geopark (6) } \\
\text { Arxan National Geopark (11) } \\
\text { Jingangtai National Geopark, Xinyang (81) } \\
\text { Fushan National Geopark (85) } \\
\text { Liuhe National Geopark (89) } \\
\text { Linhai National Geopark (93) } \\
\text { Yandangshan National Geopark (94) } \\
\text { Zhangzhou Littoral Volcanic Geomorphy } \\
\text { National Geopark (103) } \\
\text { Tengchong Volcano and Geotherm National } \\
\text { Geopark (125) } \\
\text { Weizhoudao Volcano National Geopark, } \\
\text { Beihai (130) } \\
\text { Xiqiaoshan National Geopark, Foshan (133) } \\
\text { Huguangyan National Geopark, Zhanjiang (136) } \\
\text { Shishan Volcano Cluster National Geopark, } \\
\text { Haikou (138) } \\
\text { Hongkong National Geopark (139) }\end{array}$ & $\begin{array}{l}\text { Volcanic landforms; volcanic lakes; volcanic lavas } \\
\text { Volcanic landforms; lava tunnels; volcanic craters; water landforms; } \\
\text { granite landforms } \\
\text { Longgang volcanoes; lava plateau; volcanic cones; Maar Lake; mineral } \\
\text { springs } \\
\text { Volcanic landforms; warm springs; granite peak forests; rock mortars } \\
\text { Volcanic landforms; ultra-high pressure metamorphism; magmatic intrusion } \\
\text { Volcanic rocks; volcanic cones; craters; cinders; lava flows } \\
\text { Shield volcanic clusters; volcanic columnar joints and hoodoos; river stone } \\
\text { layers } \\
\text { Late Cretaceous volcanic rocks and landforms; columnar joints; } \\
\text { pterosaurs and bird fossils } \\
\text { Early Cretaceous huge calderas; volcanic eruption remains; caldera } \\
\text { collapse; resurgence and recruption; volcanic peaks and canyons; waterfalls } \\
\text { Coastal volcanic lavas; basalt columnar joints; volcanic fumaroles and } \\
\text { cones } \\
\text { Modern volcanic landforms; various warm and hot springs; sinters } \\
\text { Volcanic craters; volcanic-sedimentary sections; coral reef } \\
\text { Volcanic cones; trachytic rocks; canyons and waterfalls; mining sites } \\
\text { and palaeoanthropogenic relics } \\
\text { Volcanic landforms; Maar Lake; lava structures } \\
\text { Basic volcanic rocks; Holocene dormant volcanoes; more than } 40 \\
\text { volcanic cones; lava tunnel; mineral springs } \\
\text { Volcanic landforms; marine erosion landforms }\end{array}$ \\
\hline
\end{tabular}


Table 1. National geoparks in China classified by major geoheritage feature or value

\begin{tabular}{|c|c|c|}
\hline Classification & Representative national geoparks (number) & Major features of geoheritage resources \\
\hline & $\begin{array}{l}\text { E-3: Glacial landforms } \\
\text { Ningwu Ice Cave National Geopark (32) } \\
\text { Kanas Lake National Geopark, Burqin (43) } \\
\text { Kunlunshan National Geopark, Golmud (47) } \\
\text { Nyanboyeshizer National Geopark, Jigzhi (49) } \\
\text { Hailuogou National Geopark (52) } \\
\text { Siguniangshan National Geopark (53) } \\
\text { Lushan Quaternary Glaciation National } \\
\text { Geopark (105) } \\
\text { Cangshan National Geopark, Dali (123) }\end{array}$ & $\begin{array}{l}\text { Glacial relics and ice caves; periglacial landforms; granite spherical } \\
\text { weathering landforms } \\
\text { Quaternary glacial relics; glacial barrier lakes; cirques, terminal moraines, } \\
\text { U-shaped valley, boulders, horns, striaes; geological tectonic relics } \\
\text { Mud-volcanic ice hummocks; palaeoglacial remnants; tectonic earthquake } \\
\text { relics } \\
\text { Modern glaciers; glacial landforms; plateau peak forest landforms } \\
\text { Modern low-altitude glaciers, horns, cirques; warm springs } \\
\text { Quaternary glacial landforms; pyramid peaks, cirques, U-shaped valleys } \\
\text { Birthpalce of Quaternary glacial research in China; integrated sections; } \\
\text { glacial relics and nominated palces; cirques, horns and U-shped valleys; } \\
\text { glacial striaes and boulders; Jiangnan ancient stratigraphic section; } \\
\text { fault-block mountains } \\
\text { Quaternary glacial relics; horns and cirques; precipitous fault depressions; } \\
\text { tectonic orogeny }\end{array}$ \\
\hline & $\begin{array}{l}\text { E-4: Fluvial landforms } \\
\text { Yellow River Delta National Geopark, } \\
\text { Dongying (26) } \\
\text { Daduhe Canyon National Geopark (59) } \\
\text { Yangtze Three Gorges National Geopark (67) } \\
\text { Yellow River National Geopark, Zhengzhou (78) } \\
\text { Chongmingdao National Geopark (91) } \\
\text { Baishuiyang National Geopark, Pingnan (97) }\end{array}$ & $\begin{array}{l}\text { Water bodies of Yellow River Delta; sedimentary features; ancient coast } \\
\text { relics; shell dykes } \\
\text { River canyons; gorges; lane valleys; Quaternary glaciers; anthropogenic } \\
\text { engineerings } \\
\text { Canyons; karst and gravity collapses; tufa deposits in karst caves; } \\
\text { landslides; stratigraphic-structural profiles; fossil sites; hazard } \\
\text { mitigation engineering. } \\
\text { Fluvial landforms; loess profiles at the top of Yellow River Delta; } \\
\text { geological engineering } \\
\text { Sedimentary features of river delta; muddy tidal flat landforms } \\
\text { Flat rocky riverbed; canyons; fluvial erosion landforms; waterfalls; } \\
\text { columnar joints }\end{array}$ \\
\hline & $\begin{array}{l}\text { E-5: Marine erosion landforms } \\
\text { Coastal Dalian National Geopark (10) } \\
\text { Changshan Isles National Geopark (25) } \\
\text { Shenhuwan National Geopark, Jinjiang (102) } \\
\text { Dapeng Peninsula National Park, Shenzhen (137) }\end{array}$ & $\begin{array}{l}\text { Marine erosion landforms; sedimentary structures; ductile shear zones; } \\
\text { geological and structural profiles; natural boundary between Yellow Sea } \\
\text { and Bohai Sea } \\
\text { Marine erosion and deposition landforms characterized by sea stacks, } \\
\text { caves, viaducts, cliffs, arches; loess deposition } \\
\text { Marine erosion landforms; lagoons; ancient oyster reefs; huge ductile } \\
\text { shear zones } \\
\text { Marine erosion and deposition landforms; palaeo-volcanic relics }\end{array}$ \\
\hline & E-6: Structural landforms & \\
\hline \multirow[t]{4}{*}{$\begin{array}{l}\text { F: Water } \\
\text { landscapes }\end{array}$} & $\begin{array}{l}\text { F-1: Springs } \\
\text { Enping Geotherm National Geopark (134) }\end{array}$ & Geothermal landscapes; granite geology and landforms \\
\hline & $\begin{array}{l}\text { F-2: Lakes and swamps } \\
\text { Xingkaihu National Geopark (4) } \\
\text { Jiuzhaigou National Geopark (56) }\end{array}$ & $\begin{array}{l}\text { Tectonic lake; lake levees; wetland; lacustrine landforms } \\
\text { Alpine lakes; springs; waterfalls; torrents; karst landforms; stratigraphic } \\
\text { profiles; fossils }\end{array}$ \\
\hline & $\begin{array}{l}\text { F-3: River } \\
\text { Yellow River Meander Bends National } \\
\text { Geopark, Yanchuan (35) }\end{array}$ & Meandering river; loess canyon landscapes \\
\hline & $\begin{array}{l}\text { F-4: Waterfalls } \\
\text { Hukou Falls National Geopark at Yellow } \\
\text { River (34) }\end{array}$ & $\begin{array}{l}\text { The biggest waterfall in the main Yangtze River; narrow and deep } \\
\text { canyons; headward erosion }\end{array}$ \\
\hline $\begin{array}{l}\text { G: Environ- } \\
\text { mental geo- } \\
\text { heritage }\end{array}$ & $\begin{array}{l}\text { Cuihuashan National Geopark of the Geologic } \\
\text { Hazard of Rock Collapse (37) } \\
\text { Yi'ong National Geopark (51) } \\
\text { Xiaonanhai National Geopark, Qianjiang (66) }\end{array}$ & $\begin{array}{l}\text { Earthquake-induced mountain collapses; barrier lake; granite } \\
\text { peak clusters; precipitous cliffs } \\
\text { Extensive mass wastings; high mountain and deep canyons; modern } \\
\text { glaciers } \\
\text { Earthquake relics; collapse and slump blocks; talus; barrier lake; karst } \\
\text { landforms; fossils; sedimentary features }\end{array}$ \\
\hline
\end{tabular}



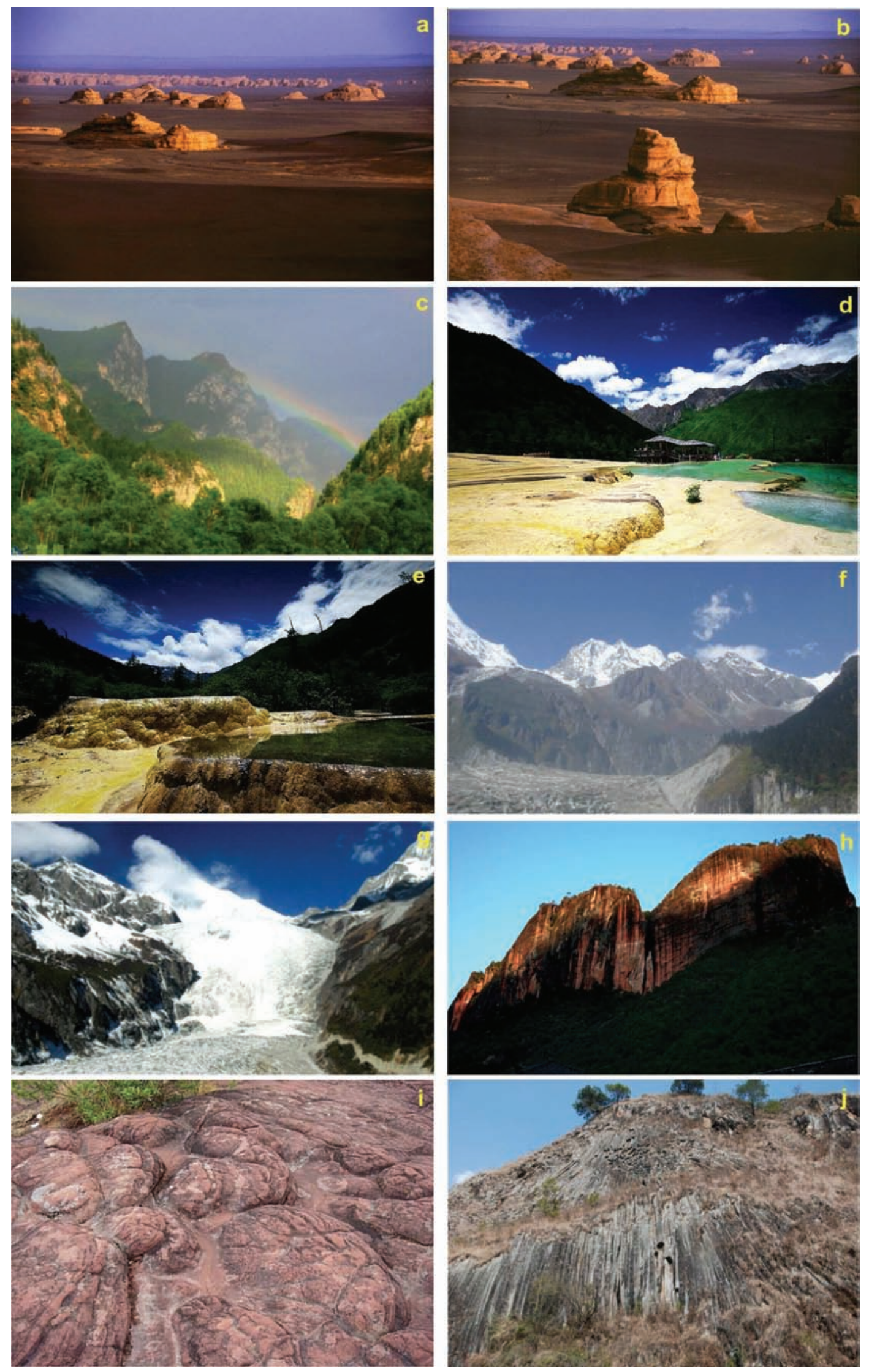

Figure 3. Examples of landform diversity in the transitional zone between the first and second geomorphic levels. Where a and b show Yardang landforms in Dunhuang; $c$ refers to karst landforms of Huzhu in Qinghai; $d$ and $e$ indicate karst landforms in Huanglong; $f$ and $g$ are the glacial landforms of Hailuogou in Sichuan; $h$ and $i$ are plateau Danxia landscapes of Yunnan; and $j$ is the volcanic landform of Tengchong in Yunnan. 

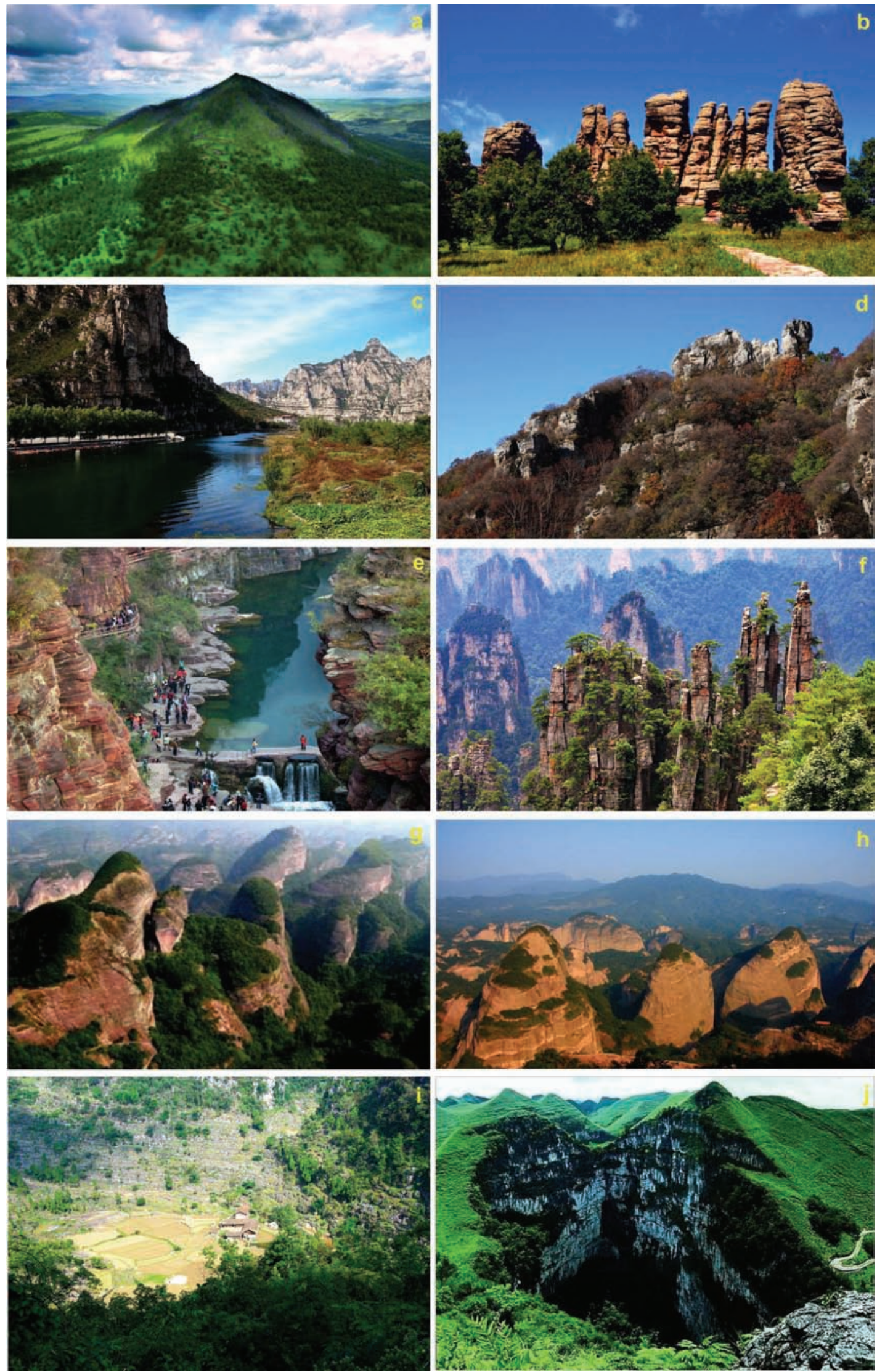

Figure 4. Various landforms in the transitional belt between the second and third geomorphic levels. Here, a shows volcanic landform in Arxan; $b$ displays granite peaks of Hexigten; $c$ is karst landform in Shidu of Beijing; $d$ is the dolomite peaks in Baishishan of Laiyuan; $e$ refers to Red Stone Valley in Yuntai Mountain; $f$ is Imperial Brush Peak in Zhangjiajie; $g$ and h are Danxia landforms at Danxia Mountain; $i$ is a karst doline at Pingtang in Guizhou; and $j$ is a doline in Baise of Guangxi. 

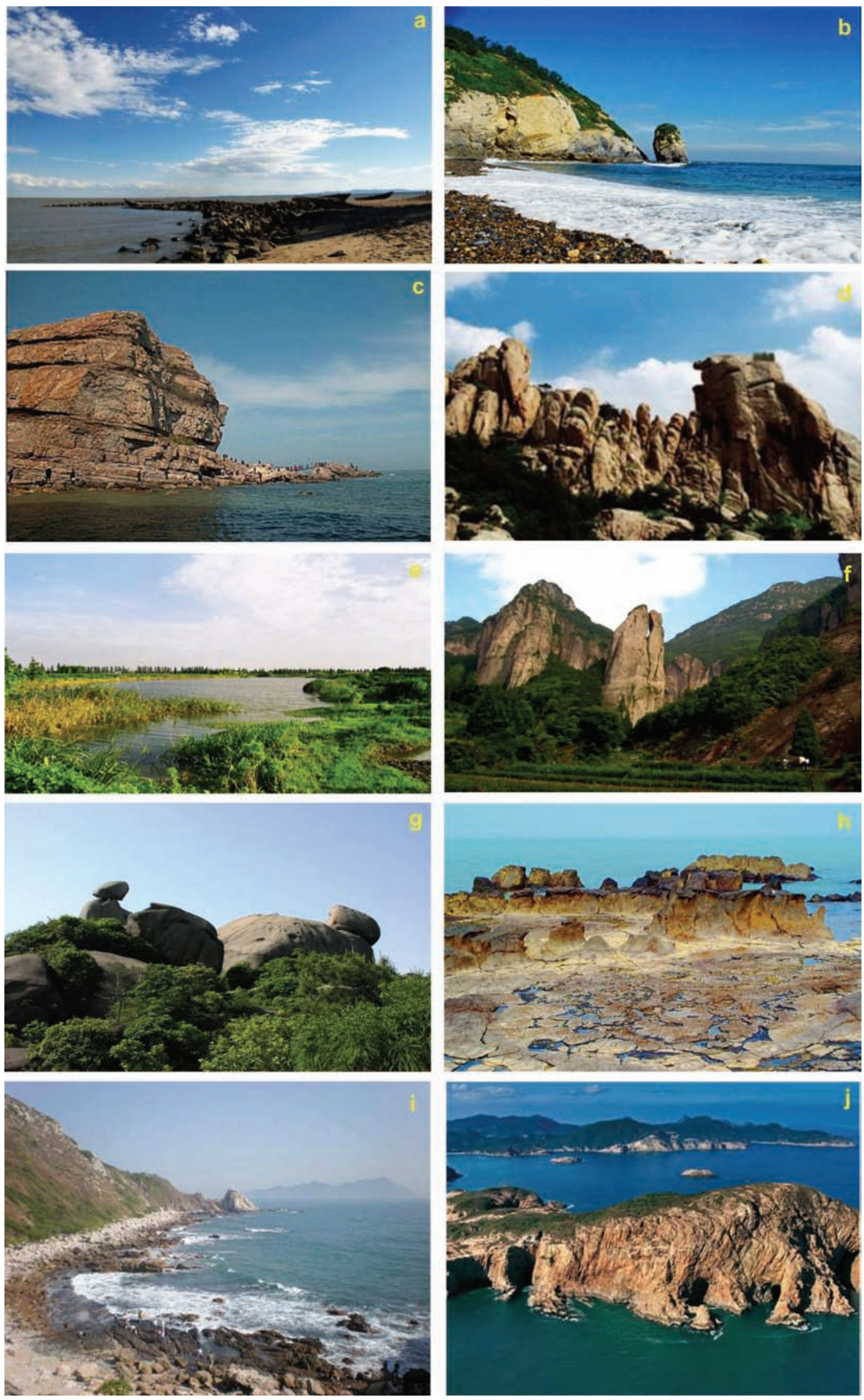

Figure 5. Different patterns in east coastline areas. Here, a is Xingkai Lake of Heilongjiang; $b$ is Dalian coastline; $c$ indicates Changdao coast of Shandong; $d$ shows granite landforms in Yimengshan; $e$ is Chongming wetland of Shanghai; $f$ and $g$ are granite landforms in Yandang Mountain; $h$ is volcanic rock in Taimushan; $i$ is coastal landforms in Dapeng peninusia in Guangzhou; $j$ is marine erosion landform in Hong Kong. 
Although many of China's national geoparks are located in regions with an annual rainfall of $>400 \mathrm{~mm}$ and annual average temperatures of $>13^{\circ} \mathrm{C}$ (Fig. 6), it is clear that climatic variations in China have nevertheless played an important role in shaping its diverse landscapes. Variations in temperature and rainfall determine weathering, transportation and deposition processes, and consequently landscape development. Landforms in the humid tropical zones of southeast China are dominated by chemical and biological weathering, with minimal mechanical weathering and abrasion. In these areas the slopes of clastic and karstic peaks and hoodoos tend to be convex, and are mantled by deeply weathered regolith (Table 1). In the temperate zones of central and north China chemical and biological weathering are not as intense as in the tropics, so that landforms tend to be sharper than those in the tropics, but less angular than those of arid lands. The arid northwestern lands and semi-arid margins are characterized by the development of bold cliffs, sharp slope breaks and a marked topographic expression of structural and lithological discontinuities, because the relatively low rainfall has minimized chemical and biological weathering and, because of a sparse vegetation cover, maximized mechanical weathering.

Active glacial/periglacial and/or frost-related processes and landforms are mainly found in the Qinghai-Tibetan Plateau areas where summits reach elevations of around 2000-3000 m (Figs. 3f, $3 \mathrm{~g}$ ). These landforms are characteristic of glacial carving (such as cirques, horns and U-shaped valleys), and steep slopes with basal accumulations of frost-shattered debris or solifluction deposits. In addition to glacial and periglacial erosion, landforms in these areas are also subjected to processes such as post-glacial stress release, salt weathering and some biochemical weathering. Differences in rock properties such as porosity, fracturing, stiffness and rock strength also affect the development of landforms in these lower temperature regimes.

\section{Lithologic control}

Rock type is also an important constraint on landform development across China, and has been emphasized by many previous investigators (Pan, 1995; Zhao and Zhao, 2003). The nature of the rock (e.g., igneous, metamorphic, clastic, carbonatic) and the degree of weathering determine the rock's resistance to erosion and therefore the development of different landscapes.

Magmatic and metamorphic rocks are exposed across a large

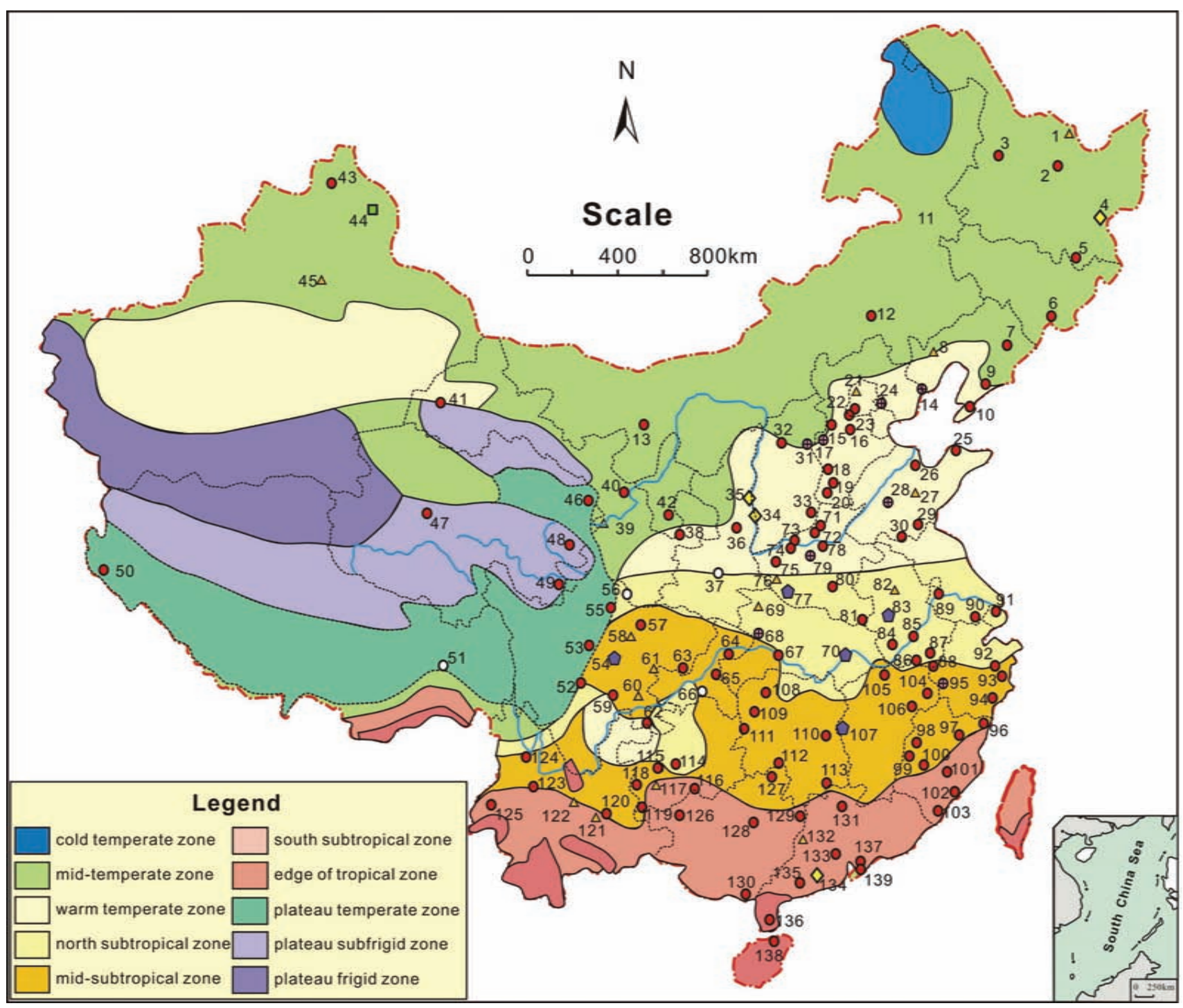

Figure 6. Distribution and variation of climatic zones across China. 
portion of China, and, because of their relatively greater resistance, often give rise to precipitous mountains and steep cliffs. Widely distributed granites, for example, give rise to steep slopes or abrupt peaks, and are well expressed at Hexigten in Inner Mongolia (Fig. 4b), Yimengshan in Shandong (Fig. 5d), Chayashan and Shenlinzhai in Henan, Tianzhushan, Huangshan and Guniujiang in Anhui, Taimushan and Shiniushan in Fujian, and Sanqingshan in Jiangxi. Quaternary basaltic lava flows are abundant in the northeast, north and southeast coastal areas, and have given rise to lava platforms in northeast China and cinder cones, lava domes, crater lakes, calderas, and other volcanic landforms in Guangxi, Guangdong, Yunnan, and Hainan in southeast China (Fig. 7).

Sedimentary rocks cover approximately $75 \%$ of China's land area and have produced some exceptional landscape features. In the southern part of the Yangtze region, terrestrial red conglomerates and coarse-grained sands deposited in humid-hot environments throughout the Mesozoic and Cenozoic periods, now constitute the base of the cliff-like Danxia terrains of Anhui, Fujian, Jiangxi, Hunan, Guangxi, and Guangdong (Figs. 3h, 3i). When affected by fluvial erosion, relatively soft sands and shales yield flat-topped low hills, mesas and buttes, such as in the red bed terrains of south China (Figs. 4g, 4h).
The widespread and thick Paleozoic carbonate rocks of the Yunnan-Guizhou Plateau, Guangxi areas and partial north and northwest China have produced remarkably spectacular karst landforms comprising hoodoos, tower karst, peak clusters, caves, and underground rivers (Figs. 3c, 3d, 3e, 4c, 4i, 4j). Those of the Guilin and World Heritage South China Karst areas are internationally famous.

Loess and aeolian landforms are primarily distributed in northwestern China, and comprise the unique loess plateau, hills and yardang landscapes of central and east Gansu, north Shaanxi and Shanxi, and Xinjiang (Figs. 3a, 3b). The extremely dry climate and abundance of loose Quaternary sediment are reflected in the vast deserts and sandy dune fields of extreme northern China.

Because of their importance and wide variability throughout China we will discuss landforms developed on sedimentary rocks in somewhat more detail.

\section{Clastic landforms}

Clastic landscapes are widely scattered across most parts of China (Huang, 1999; Peng, 2007; Qi et al., 2005; Fig. 8). Based on tectonic

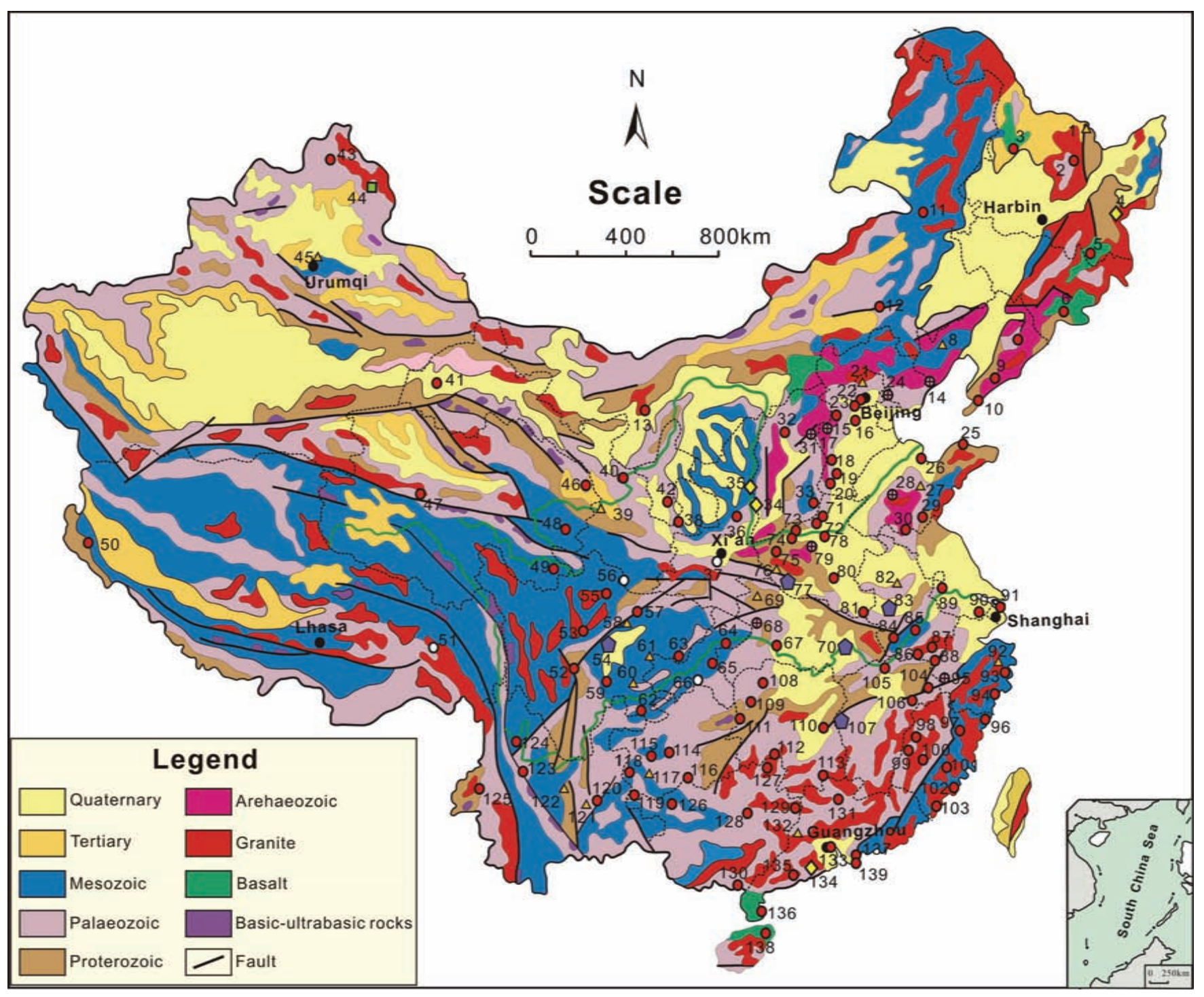

Figure 7. Geological sketch map of China indicating the ages of bedrock and the distribution of igneous rocks (modified from Ma, 2002). 


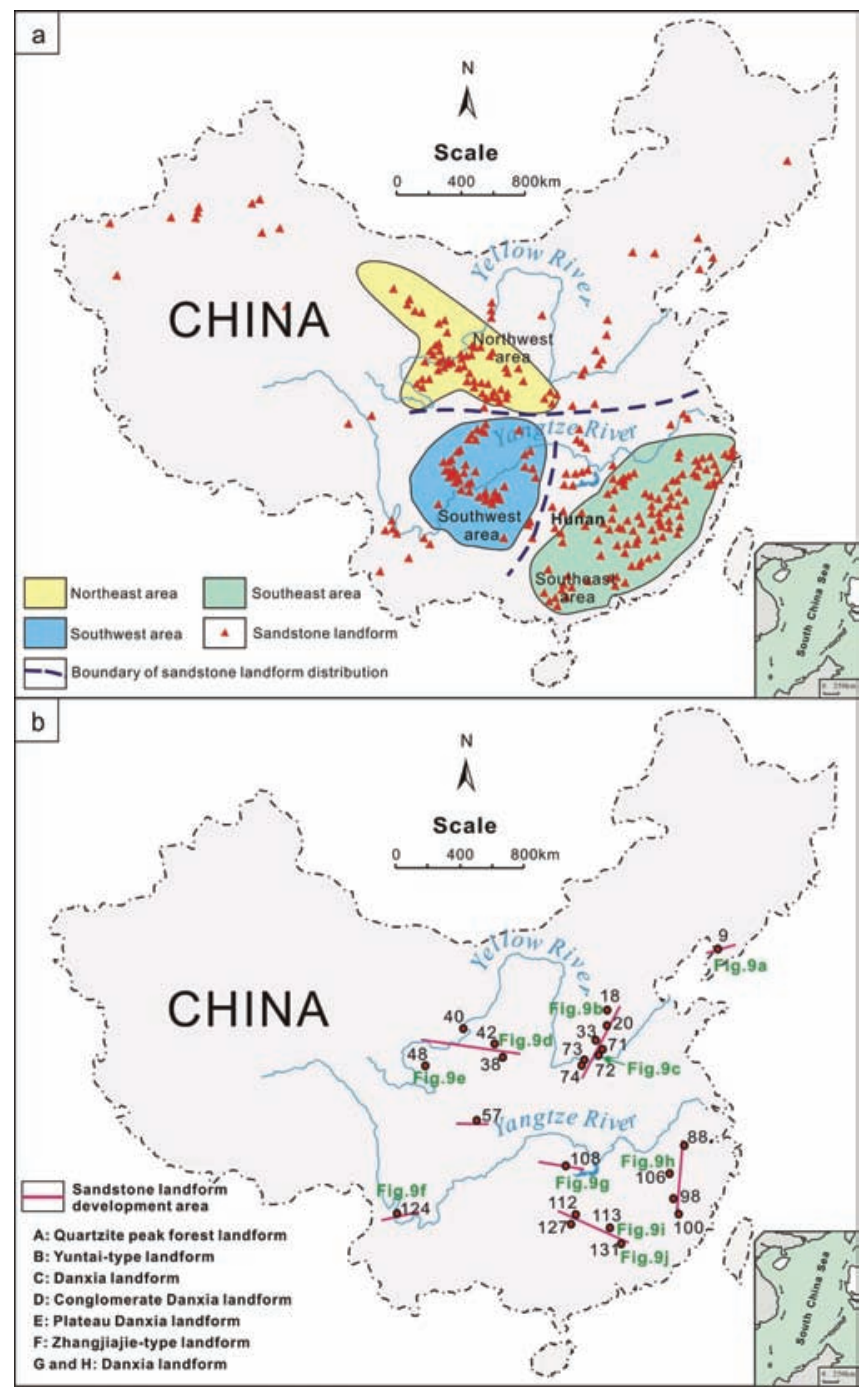

Figure 8 (a) Distribution of major clastic landforms in China (modified from Huang, 1999; Qi et al., 2005); (b) Localities of national geoparks with clastic landforms in China.

uplift rate, annual mean precipitation, vegetation cover, and areal distribution, Qi et al. (2005) and Cui et al., (2007) recognized 3 distinct areas of clastic landforms - Southeast, Southwest, and Northwest areas (Figs. 8, 9). In the Southwestern area high precipitation and active tectonic uplift tends to have favored formation of large scale canyons, steep escarpments (Fig. 9f), and large waterfalls, whereas the plentiful rainfall but minor recent uplift characteristics of the Southeastern area have promoted the formation of peak-group, and isolated peak landforms (Figs.9g, 9h, 9i, 9j). Less mature landforms are found in the cold-dry northwestern areas (Figs. 9d, 9e) and locally in northeast and north China (Figs. 9a, 9b, 9c).

\section{Karst landforms}

Because of its wide range of climatic zones China contains a remarkable diversity of karst landscapes that constitute a unique natural heritage (e.g., Luo et al., 2006; Wei et al., 2008). In contrast to other landscapes, variability among karst landscapes is a stronger function of climatic variation, and to a lesser extent lithologic characteristic, than of tectonic activity. Karst topography in eastern China varies with latitude from tropical to subtropical to temperate
(Shao et al., 2007; Wei et al., 2008; Figs. 10, 11, and 12), whereas the karst landscapes in western China and on the high Qinghai-Tibetan Plateau are characteristic of arid environments.

Tropical karst peaks are chiefly located in Guangxi, western Guangdong, eastern Yunnan and South Guizhou (Figs. 10 and 12), with Koho, hoodoos, tower karst, peak clusters and huge caves being dominant in these regions (Figs. 11i, 111). The limestone peaks typically have very steep slopes and an average peak cluster density of 2.5 per square kilometer, indicating the primary controls of many surface depressions (dolines) and many large caves and underground river systems.

Subtropical karst landscapes, located in the south flank of the Qinling Range and the Huaihe River, are characterized by gentle hills and depressions (Figs. 11g, 11h, 11j, 11k, and 12). Underground rivers in these areas are shorter than those in tropics, and there are less depressions and more dry valleys than the tropical karst areas. Positive topography is subdued, with low gentle hills with an average slope of around 25 degrees. Solution features are less well developed that in the tropical areas, and consist mostly of fissure- and arch-shaped caves.

Dry valleys and remnants of ancient flat hills are subtle characteristics of the temperate-zone karst landscapes, which are otherwise similar to non-karst landscapes (Figs. 11a, 11b, 11c, 11d, and 12). Caves are typically small fissure caves, however springs with large catchment areas and significant flow can be common, as evidenced by Baotu Spring and Niangziguan Spring. Strong downcutting of rivers in some areas, such as the Juma River in Beijing, have produced peak forest landscapes (Fig. 11c).

Karstification in arid or semiarid zones is more poorly developed, and is chiefly restricted to weathering of limestone along a few cracks (Fig. 11e). Because there are few well-developed cave systems, there is no foundation instability and surface doline depressions do not occur (Fig. 12). Plateau karst landforms in periglacial areas are largely controlled by intense freeze-thaw weathering, and this may give rise to remarkable and unique karstification processes, such as stone hills (Fig. 11e), stone walls, accumulated travertine landscapes (Fig. 11f), some shallow caves in the periglacial slopes, and occasional karst depressions.

\section{Distribution of national geoparks in China in relation to regional geology}

As previously described, many of China's national geoparks are located in the transitional areas between different geomorphic levels (Figs. 1 and 2); for instance, the southeast edge of the Tibetan Plateau is the location of the Yardang landforms of Dunhuang in Gansu and Kanbula in Qinghai, of the plateau karst landforms of Huzhu in Qinghai (Fig.3c) and Huanglong in Sichuan (Fig. 3d, 3e), and of the glacial landforms in Qinghai, Sichuan and Yunan (Figs. 1, 3f, 3g). Volcanic landscapes occur in southern Yunan Province (Fig. 3j), and volcanic and granitic landforms (Fig. 4a, 4b), as well as clastic and karst landscapes, are often concentrated in the transitional region between the second and third geomorphic levels (cf. Fig. 3h, 3i, 4c4j). Fluvial landscapes, wetland, and coastal erosional landscapes develop in the adjoining areas, and extend from the third step level to the eastern coastlines (Fig. 5a-5j).

The three latitudinal tectonic belts also control the distribution of geoheritage resources, with the central and southern belts being 

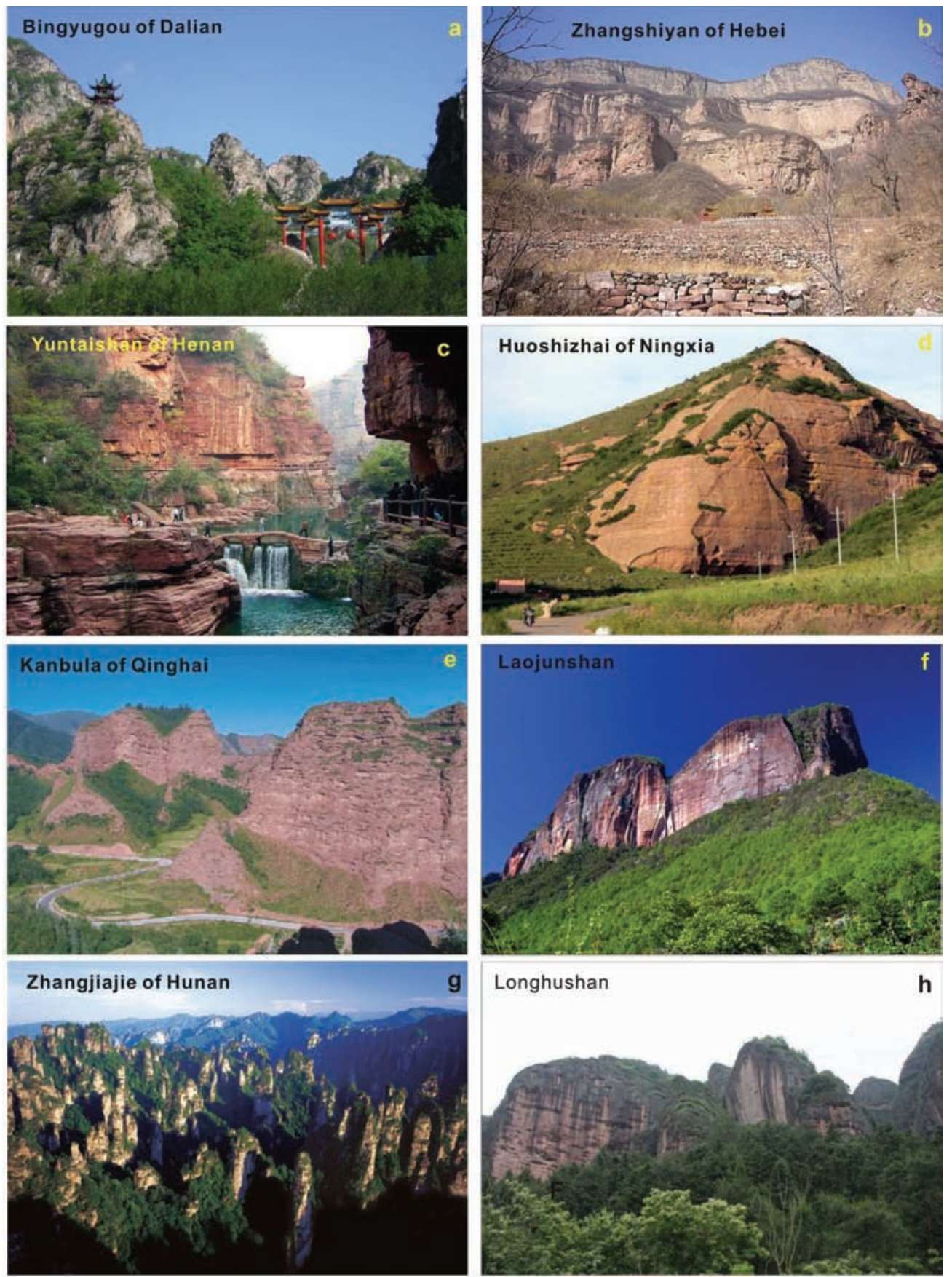

Feitianshan of Hunan

i
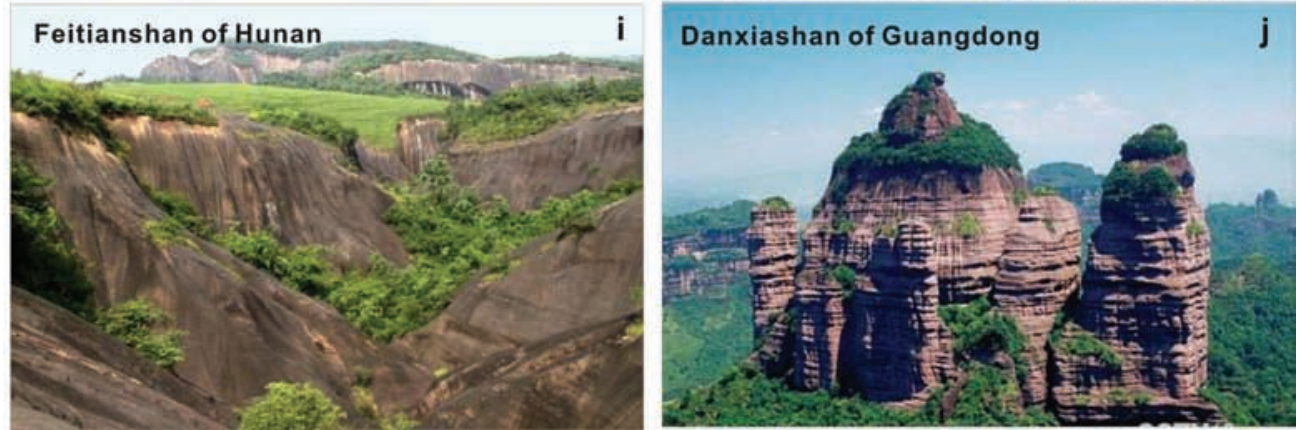

Figure 9. Major clastic landforms in China with reference to the national geoparks featured by clastic landforms as indicated in Figure 8. Here, $a$ is sandstone landform in Bingyugou of Dalian, Liaoning; $b$ is quartz sandstone landscape of Yuantaishan, Henan; $c$ indicates clastic landforms in Zhangshiya of Hebei; $d$, e, and f are the typical Danxia landforms in Huoshizhai of Ningxia, Kanbula of Qinghai, and Laojunshan of Yunan, respectively; $g$ indicates the quartz sandstone landform in Zhangjiajie of Hunan; $h$, ind $j$ are the Danxia landforms occurred in Longhushan of Jiangxi, Feitianshan of Hunan, and Danxiashan of Guangdong, respectively. 


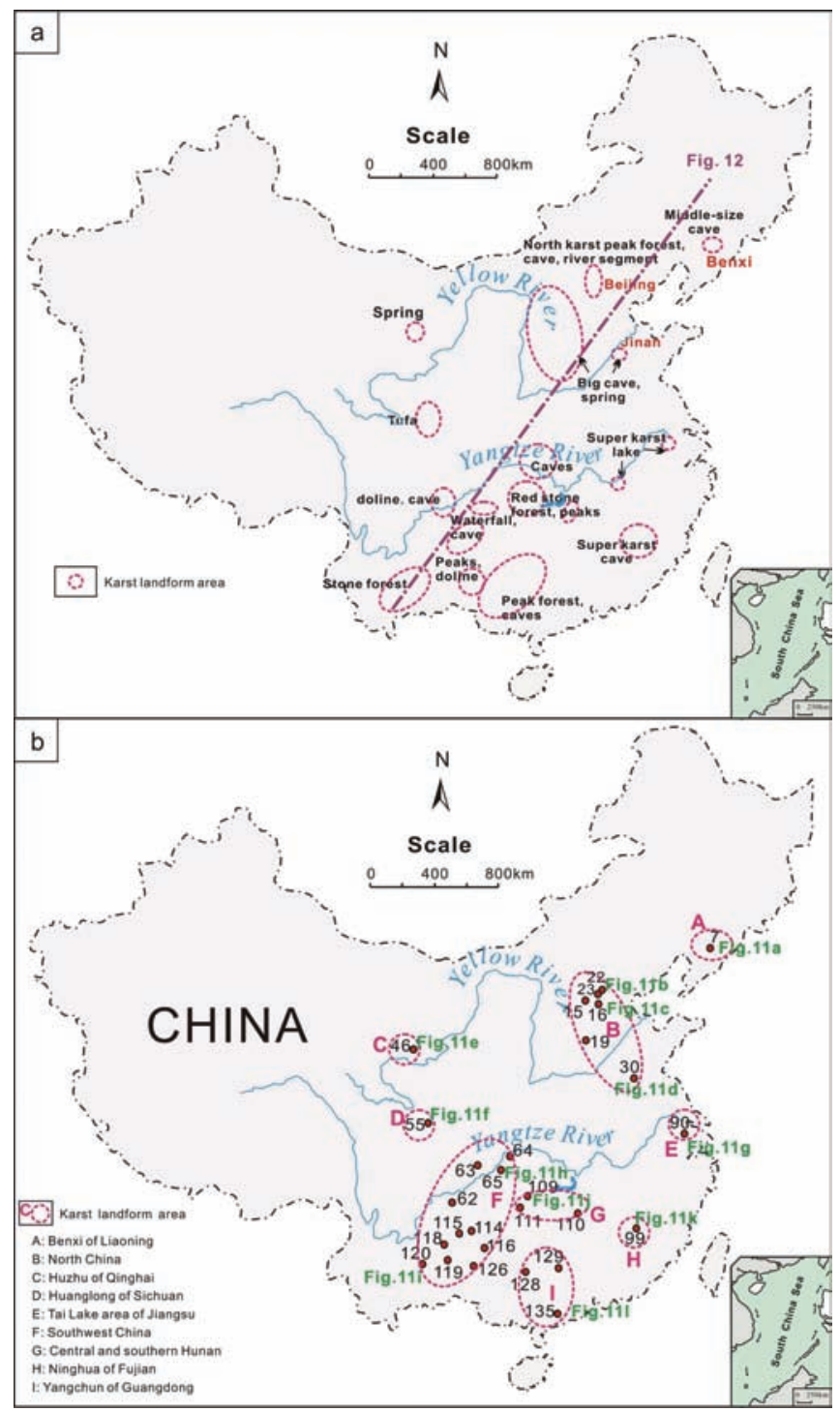

Figure 10. (a) Distribution of the major karst landform areas in China (modified from Wei et al., 2008) and showing the transect illustrated in Figure 12; (b) Localities of national geoparks with karst landforms in China.

particularly rich in geoheritage resources. For instance, the middle Qinling-Kunlun tectonic belt, roughly between latitudes $32^{\circ} \mathrm{N}$ to $35^{\circ} \mathrm{N}$, is the setting for notable mountain ranges that include the Danxia landforms of Kanbula in Qinghai, for environmental geoheritage sites in Cuihuashan (Shaanxi), and for type stratigraphic and tectonic sections in Henan. The southern tectonic belt, at latitudes between $23^{\circ} 30^{\prime} \mathrm{N}$ and $26^{\circ} 30^{\prime} \mathrm{N}$, includes spectacular Danxia landforms in Liangshan and Feitianshan (Hunan), Ziyuan (Guangxi), and Renhua (Guangdong).

Clastic and karst landforms are widely distributed throughout the country with significant regional variability. The most important clastic landform areas in China occur in the southeast, northwest and north. The karst landscapes in central China are of modest extent, especially when compared to those of southwest China, but they display important caves and other solution features. Glacial landforms are primarily distributed in the southeast margin of Qinghai-Tibetan Plateau, including geoparks 47, 49, 52, 53, 123 and partial 124. Palaeontologic geoheritage resources are primarily concentrated in
Yunan, Sichuan, and Guizhou, and locally in Xijiang, Beijing, Henan, Hubei, Zhejiang and also Northeast China. Coastal erosion and island landscapes concentrate along the eastern coastline.

\section{Geographical distribution of national geoparks in China}

Geoheritage resources in China can be divided into 7 regions on the basis of geological-geomorphic features as well as local characteristics of socio-economic development (Fig. 1 and Table 2).

Zone I covers most of Northeast China and eastern Inner Mongolia and is characterized by volcanic landforms, as well as Quaternary glacial and periglacial features. Volcanic cones, craters, volcanic lakes, and coarse pyroclastic ejecta are widely distributed in eastern Inner Mongolia, Heilongjiang, and Jilin Changbai Mountains (Fig. 4a). Remains of quaternary glaciers, ice sheets, permafrost, and lakes (Fig. 5a) are scattered throughout the Songnen Plain, southwest Heilongjiang.

Because of the relatively stable North China platform, Zone II, which includes much of North China and southern Northeast China, preserves fairly complete stratigraphic sections and valuable paleontological remains. These include the Middle-Late Proterozoic type section in Tianjin, the Cambrian section in Shandong, Mesozoic Jehol biotas in western Liaoning and eastern Inner Mongolia, dinosaur-egg fossils in Henan, and the famous Peking Man Site at Zhoukoudian near Beijing. Coastal landforms in these areas preserve features of marine erosion and island landforms, such as in Golden Pebble Beach in Dalian and Changdao (Fig. 5b, 5c).

Zone III corresponds to Central China and the coastal areas of South China. This zone includes the provinces of Hunan, Hubei, Jiangxi, Jiangsu, Shanghai, Zhejiang, Fujian, Guangdong, Hainan and southern Anhui. The chief geological heritage resources in this zone are the Danxia red sandstone landforms, coastal landforms and volcanic landscapes (Figs. 4f-4j, 5e-5j). The spectacular Danxia landforms, incised in Mesozoic red sandstone, are largely the result of erosion in a humid climate with abundant rainfall. Coastal landforms exemplify marine geological processes. Volcanic landforms occur in Yandang Mountain (Zhejiang) and Huguangyan (Guangdong).

Zone IV encompasses the provinces of Guizhou, Chongqing, Guangxi, Yunan and most of Sichuan in Southwest China in which the most important geoheritage resources are fossil localities and karst terrains. Paleontological remains in this area are varied, including the Cambrian Kaili fauna of Jianhe in Guizhou, the Cambrian Chengjiang fauna of Yunnan, Mesozoic vertebrates, chiefly marine reptiles and dinosaurs in Xingyi and Guanling (Guizhou), Zigong (Sichuan), and Lufeng (Yunan). The hot and humid climate has combined with carbonate sequences to give rise to widespread karst landscapes, including karst peaks, towers, caves, canyons, dolines, and travertine springs, terraces and waterfalls (Fig.3d, 3e).

The Shaanxi-Gansu Loess Plateau defines Zone V, and includes most of Shaanxi and Gansu provinces. This area is characterized by arid climates and from a geoheritage perspective is the worldwide type-locality for loess landforms and related fluvial landscapes. River erosion is responsible for the generation of various loess landforms, such as loess mesas, ridges, hills, ditches and columns in Luochuan of Shaanxi. The Yellow River has actively changed course in historical times, and has given rise to river terraces, large meandering river systems and other fluvial landforms, chiefly in Yanchuan (Shaanxi). 

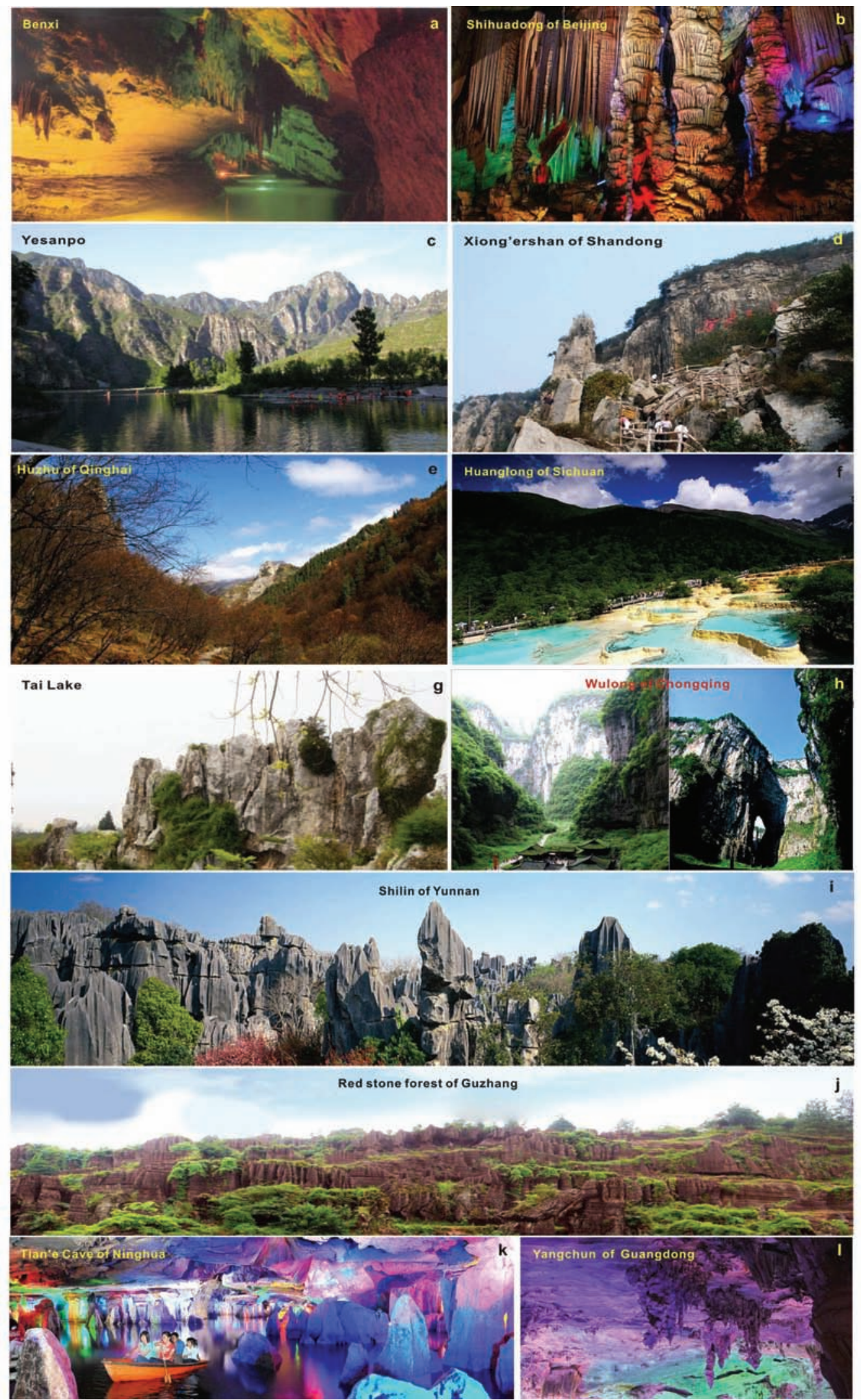

Figure 11. Major karst landforms in China with reference to the national geoparks characterized by karst landscapes indicated in Figure 10. Note that, a is karst landform in Benxi Water Cave of Liaoning; b, c, and d are the karst landscapes in Shihuadong of Beijing, Yesanpo of Hebei, and Xiong'ershan of Shandong, respectively; $e$ and $f$ are the typical karst landforms in Huzhu of Qinghai and Huanglong of Sichuan; $g$ is karst topography in Tai Lake of Jiangsu; $h$ and $i$ are the representative karst landscapes in Wulong of Chongqing and Shilin of Yunnan; $j$ shows the karst landform in Guzhang of Hunan; $k$ represents the Tian'e Cave in Ninghua of Fujian; l displays the karst cave in Yangchun of Guangdong. 


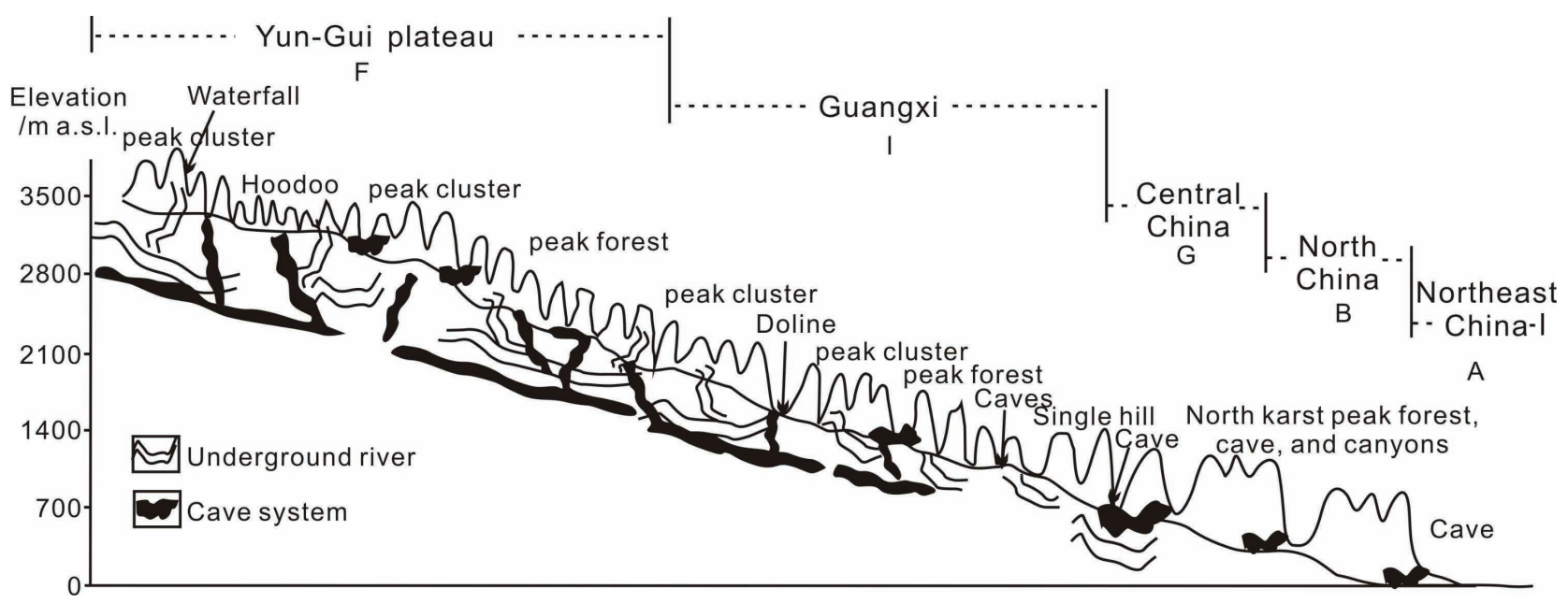

Figure 12 Simplified development patterns of karst landforms from southwest Yun-Gui Plateau to northeast China along the transect indicated on Figure 10a (modified from Wei et al., 2008).

Zone VI covers the Northwest China, including Xinjiang, western Inner Mongolia, Ningxia and western Gansu. The most important geological heritage resources are aeolian landforms, including Yardang landforms in Dunhuang (Fig.3a, 3b), Gansu Province, high mountain valleys and the glacial landforms in Tianshan. Fossils are also abundant, and include silicified wood in Qitai and dinosaur fossils in Gansu.

The Plateau Area of Tibet, Qinghai and western Sichuan correspond to Zone VII, and the geoheritage includes glacial landforms, mountains and canyons, and salt lakes. The area includes many active glaciers in Tanggula, the Himalayas and other mountain ranges. It also includes the Brahmaputra Grand Canyon and Qinghai
Lake. Intensive Cenozoic Himalayan tectonics is responsible for the formation of the Shigatse ophiolite belt in Tibet.

\section{Concluding remarks}

The recent designation of many new geoparks in China has renewed local and more recently international interest in China's varied range of landforms. The nation-wide overview of national geoparks presented here shows that the diversity of geoheritage resources is a good representation of the natural environments and landscape forming processes (functions of tectonics, climate and rock characteristics) found throughout China. Many national geoparks are

Table 2 Zonation description of geoheritage resources in China by geoheritage type of primary geoheritage resource

\begin{tabular}{|c|c|c|c|}
\hline No. & Zoning & Major regions & $\begin{array}{l}\text { Primary geoheritage resources (serial number } \\
\text { of typical National Geopark(s) ) }\end{array}$ \\
\hline $\mathrm{I}$ & $\begin{array}{l}\text { Most parts of Northeast } \\
\text { China and eastern Inner } \\
\text { Mongolia }\end{array}$ & $\begin{array}{l}\text { Heilongjiang, Jilin, north Liaoning } \\
\text { and Eastern Inner Mongolia }\end{array}$ & $\begin{array}{l}\text { 1) Volcanic landforms (11) } \\
\text { 2) Glacial and frozen remains (9) }\end{array}$ \\
\hline II & $\begin{array}{l}\text { North China and southern } \\
\text { Northeast China }\end{array}$ & $\begin{array}{l}\text { Southeast and west Liaoning, Beijing, Tianjin, } \\
\text { Hebei, Shanxi, Shandong, most parts of Henan, } \\
\text { southeast Inner Mongolia and northern Anhui. }\end{array}$ & $\begin{array}{l}\text { 1) Stratigraphic section }(24) \\
\text { 2) Paleontology }(8,30,76) \\
\text { 3) Marine geoheritage }(10,95)\end{array}$ \\
\hline III & $\begin{array}{l}\text { Central China and coastal } \\
\text { areas of Southern China }\end{array}$ & $\begin{array}{l}\text { Hunan, Hubei, Jiangxi, Jiangsu, Shanghai, } \\
\text { Zhejiang, Fujian, Guangdong, Hainan and } \\
\text { southern Anhui }\end{array}$ & $\begin{array}{l}\text { 1) Danxia landform }(88,98,100,106,112,113,127,131 \text {, } \\
\text { 2) Coastal landform }(137) \\
\text { 3) Volcanic landform }(93,94,103,133,136,138)\end{array}$ \\
\hline IV & Southwest China & Guizhou, Guangxi, Yunnan and most Sichuan & $\begin{array}{l}\text { 1) Palaeontology and palaeoanthropology }(58,60,61,117 \text {, } \\
\text { 119, 121, 122) } \\
\text { 2) Karst landscapes }(55,62,63,114,115,118,119,120 \text {, } \\
126,128,129)\end{array}$ \\
\hline $\mathrm{V}$ & $\begin{array}{l}\text { Shaanxi-Gansu Loess } \\
\text { Plateau }\end{array}$ & $\begin{array}{l}\text { Most of Shaanxi, Gansu, eastern and } \\
\text { western Shanxi }\end{array}$ & $\begin{array}{l}\text { 1) Loess landform, including plateau, beam, hilly, ditch, } \\
\text { loess column ( } 36) \\
\text { 2) Fluvial and waterfall landforms }(34,35) \\
\text { 3) Canyon }(33,40)\end{array}$ \\
\hline VI & Northwest China & $\begin{array}{l}\text { Xinjiang, western Inner Mongolia, } \\
\text { Ningxia and Western Gansu }\end{array}$ & $\begin{array}{l}\text { 1) Aeolian landforms }(13,41) \\
\text { 2) Canyon } \\
\text { 3) Glacial landforms }(12,43) \\
\text { 4) Palaeontology and palaeoanthropology }(39,45)\end{array}$ \\
\hline VII & Tibetan Plateau & Tibet, Qinghai and western Sichuan & $\begin{array}{l}\text { 1) Glacial landform }(47,49,52,53) \\
\text { 2) Canyon (59) }\end{array}$ \\
\hline
\end{tabular}


located within the transitional areas between the countries west to eastward declining geomorphic level, and their distribution is also controlled by latitudinal tectonic belts. Regional climatic variations are significant throughout China, and these clearly are also a critical factor in the variability of landform development. Lithological variability is also seen to be important. Because of their wide distribution, clastic and karst landforms are of particular interest to Chinese geomorphologists, and have thus far been the most internationally publicized of the Chinese geoheritage. However it is very clear that a vast range of important geoheritage sites are very common within China, and much is now being done within China to recognize, protect and manage these locations as National and Global Geoparks.

\section{Acknowledgements}

We acknowledge valuable comments provided by Professor Franz Wolfgang Eder. We greatly benefitted from discussions on national geoparks with Professor Alberto E. Patiño Douce. We thank anonymous reviewers for helpful critical comments.

\section{References}

Cui, Z.J., Zhang, W., Chen, Y.X., 2007, Comparative observation of sandstone landform in China and US: implication for Danxia landscape. Proceedings of International Forum on Yuntai Landform: Interpretation and Sustainable Development, pp. 5459 (in Chinese).

Eder, F.W., 1999. UNESCO Geoparks: a new initiative for protection and sustainable development of the earth heritage. N.Jb.Geol.Paleont., Abh., Nov.1999, 214(1/2): 353- 358.

Eder, F.W., Patzak, M., 2004, Geoparks-geological attractions: A tool for public education, recreation and sustainable economic development. Episodes v. 25, pp. 162-164.

Huang, J., 1999, On the distribution of Danxia landforms in China. Economic Geography, v. 19 (suppl.), pp. 31-35 (in Chinese).

Leman, M.S., Reedman, A., Pei, C.S., 2008, Geoheritage of East and Southeast Asia. Ampang Press SDN. BHD., pp. 1-308.

Li, L.R., Jiang, J.J., Wang, W., 2002, Geoheritage resources and their management of China. China Land Press, Beijing (in Chinese).

Liu, J.Q, 1999, Volcanoes of China. Science Press, Beijing, pp 1-219 (in Chinese).

Luo, H., Chen, J.T., Zhong, G.P., 2006, A comparison between the tourism scenery of Danxia and karst landforms. Tropical Geography v.26, pp.12-17 (in Chinese).

Ma, L.F., 2002. Geological Atlas of China. Geological Publishing House, Beijing (in Chinese).
Ministry of Land \& Resources of the People's Republic of China, 2004, The world geoparks of China. China Environmental Science Press, Beijing, pp. 1-141 (in Chinese).

Pan, J., 1995, World culture and nature heritage of China. Geological Publishing House, Beijing, pp. 88-125 (in Chinese).

Peng, H., 2007, The Red Stone Park of China, Danxiashan. Geological Publishing House, Beijing (in Chinese).

Qi, D.L., Yu, R., Zhang, R.S., Ge, Y.J., Li, J.L., 2005, Comparative studies of Danxia landforms in China. Journal of Geographical Sciences, v.15, pp.337-345.

Shao, J.A., Li, Y,B., Wang, S.J., Wei, C.F., Xie, D.T., 2007, The distribution and diversity of landscape patches in different lithology-landform types in karst mountain areas. Journal of Natural Resources v.22, pp.478-485 (in Chinese).

Sun, Y.L., 2006, Assessment of georelics and suggestions for sustainable development in national geoparks. Master thesis of Guizhou Normal University, pp. 1-45 (in Chinese).

UNESCO, 2008, Operational Guidelines for the Implementation of the World Heritage Convention.

UNESCO, 156Ex/11Rev, 1999, UNESCO Geoparks Programme: a new initiative to promote a global network of Geoparks safeguarding and developing selected areas having significant geological features. Paris.

Wei, Y.L., Chen, W.H., Huang, B.J., Qin, J.,X., 2008, Spatial pattern of karst tourism resources in China. Journal of Guilin University of Technology, v.28, pp. 473-483 (in Chinese).

Wu, S.M., 2005, Geological book of China: records from 21 national geoparks. Shandong Pictural Publishing House, Jinan. (in Chinese)

Wu, Z.H., 2001, Cenozoic tectonic-morphologic evolution and mechanism of continental China and its adjacent areas. Geological Publishing House, Beijing, pp.1-275 (in Chinese).

Yang, G.F., Zhang, X.J., Tian, M.Z., Brierley, G.J., Chen, A.Z., Ping, Y.M., Ge, Z.L., Ni, Z.Y., 2011, Alluvial terrace systems in Zhangjiajie of northwest Hunan, China: implications for climatic change, tectonic uplift and geomorphic evolution. Quaternary International, v. 233, pp. 27-39.

Zhao, T., Zhao, X., 2009, Geoheritage taxonomy and its application. Acta Geoscientica Sinica, v.30, pp.309-324 (in Chinese).

Zhao, X., Wang, M., 2002, National geoparks initiates in China: Putting geosciences in the service of society. Episodes, v.25, pp. 33-37.

Zhao, X., Zhao, T., 2003, Geological background of national geoparks of China and construction of world geoparks. Geological Bulletin of China, v.22, pp.620-630 (in Chinese).

Zouros, 2002, The European Geoparks Network: Geological heritage protection and local development. Episodes, v. 25, pp.165-171. 


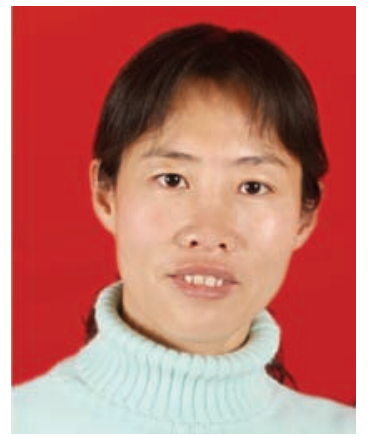

Guifang Yang is Associate Professor in Geomorphology and Quaternary Geology at China University of Geosciences, Beijing (CUGB). She got her $\mathrm{PhD}$ in Quaternary Geology with a thesis on Paleoclimatic reconstruction at China University of Geosciences, Wuhan in 2003. Since 2005, she serves as professional staff at China University of Geosciences, Beijing and coordinator of Chinese projects of Geomorphology and Quaternary Paleoenvironment. Her other principal professional interest is improving public awareness of earth science by making relevant geoscience information available to students and the general public.

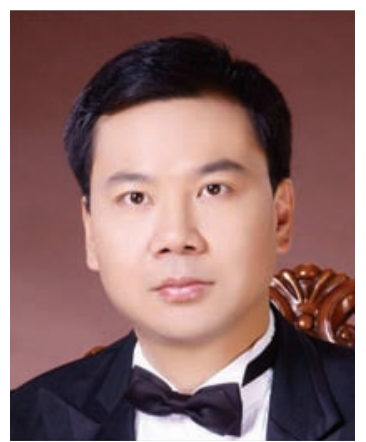

Zhenghong Chen obtained his $\mathrm{PhD}$ in Philosophy of Science and Technology from Beijing Normal University in 2009. Currently he is working as an Assistant Professor at Development Research Center, China Meteorological Administration (CMA). He specializes in meteorological hazards research and works for Development Research Center at CMA. His current research includes policy assessment of meteorological science and technology and enhancement of meteorological emergency management.

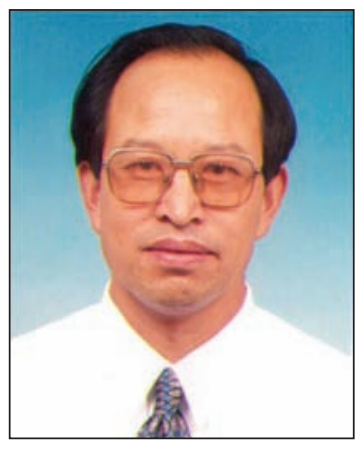

Mingzhong Tian is full Professor in Geomorphology and Quaternary Geology from China University of Geosciences, Beijing (CUGB). He is a leader of several Chinese projects related to geomorphology and Geopark construction. He has published over 80 papers and more than 10 books ranging from Quaternary geology, geomorphology, geopark planning and management.

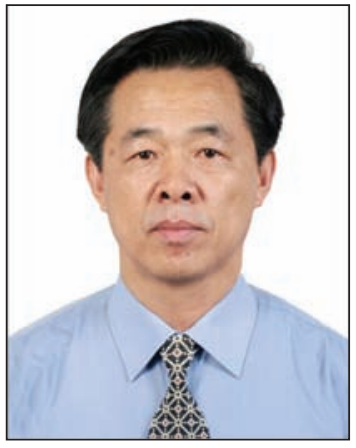

Fadong Wu is a full Professor in Sedimentology and Quaternary Geology from China University of Geosciences, Beijing (CUGB). He is a leader of Chinese projects concerned with Geopark construction and management, Technique Consultant of various national geoparks in China. His research interests span a range of topics within applied geomorphology, Quaternary paleoclimatology, and sedimentology. He has authored/ coauthored over 60 publications and several books covering sedimentology, geopark planning and management. 